\title{
An Introduction to Multiobjective Simulation Optimization
}

\author{
SUSAN R. HUNTER, ERIC A. APPLEGATE, VIPLOVE ARORA, and BRYAN CHONG, \\ Purdue University \\ KYLE COOPER, Purdue University and Tata Consultancy Services \\ OSCAR RINCÓN-GUEVARA and CAROLINA VIVAS-VALENCIA, Purdue University
}

\begin{abstract}
The multiobjective simulation optimization (MOSO) problem is a nonlinear multiobjective optimization problem in which multiple simultaneous and conflicting objective functions can only be observed with stochastic error. We provide an introduction to MOSO at the advanced tutorial level, aimed at researchers and practitioners who wish to begin working in this emerging area. Our focus is exclusively on MOSO methods that characterize the entire efficient or Pareto-optimal set as the solution to the MOSO problem; later, this set may be used as input to the broader multicriteria decision-making process. Our introduction to MOSO includes an overview of existing theory, methods, and provably convergent algorithms that explicitly control sampling error for (1) MOSO on finite sets, called multiobjective ranking and selection; (2) MOSO with integer-ordered decision variables; and (3) MOSO with continuous decision variables. In the context of integer-ordered and continuous decision variables, we focus on methods that provably converge to a local efficient set under the natural ordering. We also discuss key open questions that remain in this emerging field.
\end{abstract}

CCS Concepts: • Mathematics of computing $\rightarrow$ Probability and statistics; Probabilistic algorithms; - Computing methodologies $\rightarrow$ Simulation theory; Applied computing $\rightarrow$ Multi-criterion optimization and decision-making;

Additional Key Words and Phrases: Multiobjective simulation optimization, stochastic multiobjective optimization

\section{ACM Reference format:}

Susan R. Hunter, Eric A. Applegate, Viplove Arora, Bryan Chong, Kyle Cooper, Oscar Rincón-Guevara, and Carolina Vivas-Valencia. 2019. An Introduction to Multiobjective Simulation Optimization. ACM Trans. Model. Comput. Simul. 29, 1, Article 7 (January 2019), 36 pages.

https://doi.org/10.1145/3299872

S. R. Hunter and E. A. Applegate were supported in part by the National Science Foundation (NSF) grant CMMI-1554144. V. Arora was supported in part by NSF grant CMMI-1549608. C. Vivas-Valencia was supported in part by the Agency for Healthcare Research and Quality (AHRQ) grant 5P30HS024384.

Authors' addresses: S. R. Hunter, E. A. Applegate, V. Arora, B. Chong, K. Cooper, and O. Rincón-Guevara, School of Industrial Engineering, Purdue University, 315 N. Grant St., West Lafayette, IN 47907; K. Cooper, Tata Consultancy Services, 1000 Summit Dr., Milford, OH 45150; C. Vivas-Valencia, Weldon School of Biomedical Engineering, Purdue University, 206 S. Martin Jischke Dr., West Lafayette, IN 47907; emails: \{susanhunter, applegae, arora34, chongb, coope149, orincong, cvivas\}@purdue.edu.

Permission to make digital or hard copies of all or part of this work for personal or classroom use is granted without fee provided that copies are not made or distributed for profit or commercial advantage and that copies bear this notice and the full citation on the first page. Copyrights for components of this work owned by others than ACM must be honored. Abstracting with credit is permitted. To copy otherwise, or republish, to post on servers or to redistribute to lists, requires prior specific permission and/or a fee. Request permissions from permissions@acm.org.

(C) 2019 Association for Computing Machinery.

1049-3301/2019/01-ART7 \$15.00

https://doi.org/10.1145/3299872 


\section{INTRODUCTION}

We consider the problem of multiobjective simulation optimization (MOSO), that is, nonlinear multiobjective optimization in which the objective functions can only be observed with stochastic error. For example, multiple simultaneous objective functions may be defined implicitly through a Monte Carlo simulation model. These problems frequently arise when computer models are used to design stochastic systems. A general formulation of this problem is

$$
\begin{aligned}
\text { Problem } S \text { : minimize } & \left(\mathbb{E}\left[G_{1}(\mathbf{x}, \xi)\right], \ldots, \mathbb{E}\left[G_{d}(\mathbf{x}, \xi)\right]\right) \\
\text { s.t. } & \mathbb{E}\left[H_{i}(\mathbf{x}, \xi)\right] \leq 0 \text { for all } i=1,2, \ldots, c, \\
& \mathbf{x} \in \mathcal{X},
\end{aligned}
$$

where $\left(\mathbb{E}\left[G_{1}(\mathbf{x}, \xi)\right], \ldots, \mathbb{E}\left[G_{d}(\mathbf{x}, \xi)\right]\right)$ is a vector of $d \geq 2$ unknown objective functions; if present, $\mathbb{E}\left[H_{i}(\mathbf{x}, \xi)\right]$ are unknown constraint functions for all $i=1,2, \ldots, c$; the feasible set $\mathcal{X}$, which may be specified by a set of deterministic constraints, is nonempty and known; and $\xi$ is a random vector with support $\Xi$, such that $\mathbb{P}\{\xi \in \Xi\}=1$. Usually, the objectives in Problem $S$ are conflicting, so that no single feasible point minimizes all of the objectives simultaneously. Rather, multiple feasible points may be efficient; that is, no other feasible point maps to an objective vector that is at least as small on all objectives, and strictly smaller on at least one objective. The collection of all efficient points is called the efficient set, which is the solution to Problem $S$. We call the image of an efficient point a Pareto point, and we call the image of the efficient set the Pareto set.

MOSO problems sit at the interface of two existing and relatively well-developed research fields, deterministic multiobjective optimization (MOO) and single-objective simulation optimization (SOSO). The MOO literature considers a version of Problem $S$ in which all $d \geq 2$ objectives can always be observed without stochastic error, while the SOSO literature considers a version of Problem $S$ with $d=1$ unknown objective function that can only be observed with stochastic error. Despite significant development in MOO and SOSO separately, the methodological and theoretical development of specialized, provably convergent MOSO algorithms remains in its infancy.

Historically, solving a SOSO problem has been considered a computationally intensive task (Fu 2002), and solving a MOSO problem usually requires even more computational effort. To solve a MOSO problem, one often must generate multiple efficient points. Sometimes, generating multiple efficient points requires solving many SOSO subproblems, each yielding a single efficient point. The recent availability of mature and efficient SOSO methods, coupled with the ubiquitous availability of parallel computing power, makes characterizing the efficient set as the solution to a MOSO problem seem like an increasingly realistic goal. Members of the Monte Carlo simulation community have expressed interest in advancing this literature (Fu et al. 2014).

To facilitate future research on MOSO, we provide an introduction to MOSO theory, methods, and provably convergent algorithms that explicitly control sampling error. We focus exclusively on methods that characterize the entire efficient set as the solution to the MOSO problem, and we emphasize convergence to a local efficient set when the feasible set is not finite. Our goal is to provide a single, cohesive starting point for researchers and practitioners who wish to begin working in this nascent area. In the remainder of this section, we discuss MOSO application areas (Section 1.1), provide an overview of related literature (Section 1.2), and describe our notational conventions (Section 1.3). Since we target our writing primarily to SOSO researchers and practitioners, throughout this article, we assume the reader is familiar with SOSO literature and write at the advanced tutorial level. For readers without this background, we provide references to tutorials and entry points to the SOSO literature in Section 1.2.2. Then, in Section 2, we formulate the MOSO problem and its solution, discuss how to evaluate MOSO algorithms, and discuss the scope of our article in detail. Sections 3, 4, and 5 contain an overview of existing MOSO theory, methods, and algorithms, categorized by the nature of the feasible set $\mathcal{X}$. Section 6 contains 
references to available software and problem testbeds. Finally, Section 7 contains open questions and concluding remarks.

\subsection{Applications}

The MOSO problem arises in a variety of application areas because its formulation as Problem $S$ is extremely general. The objective and constraint functions can be defined implicitly, such as through a Monte Carlo simulation model, thus embedding nearly any level of system complexity. MOSO problems may arise as a part of multicriteria decision making (MCDM) or as part of a method used to solve other optimization problems. We begin with three such example problems.

Example 1.1. Cadaveric organ transplants are potentially life-saving resources for many patients. Organ allocation policies, such as the policies for allocating cadaveric livers and kidneys, often are evaluated using Monte Carlo simulation (Bertsimas et al. 2013; Feng et al. 2013). Such policies require the consideration of multiple objectives, including system-wide measures such as expected total patient deaths while waiting for transplantation (minimize), expected total qualityadjusted life year gains (maximize), and a measure of expected "fairness" in organ transplantation (maximize, or pose as a constraint) (Akan et al. 2012; Bertsimas et al. 2013). Feng et al. (2013) use Monte Carlo simulation to evaluate liver allocation policies with respect to five such system-wide objectives.

Example 1.2. MOSO problems arise in the design of efficient power plants, specifically, in the design of an "advanced coal-fired, gasification-based, zero-emissions electricity and hydrogen generation facility with carbon capture" (Subramanyan et al. 2011, p. 2667). The objectives detailed in the power plant design case study of Subramanyan et al. (2011) include a measure of the expected overall efficiency of the process (maximize), the expected carbon dioxide emissions (minimize), and the expected solid oxide fuel cell current density (maximize).

Example 1.3. Plant breeders often are concerned with the mating design problem, one version of which can be stated as follows. Given a population of parent plants and a finite budget of progeny plants to grow in the field, how many progeny plants should be bred from each potential parent pair to maximize the expected maximum of a particular trait, such as yield, in the progeny population? For normal trait distributions, Hunter and McClosky (2016) prove that one should only breed from parent pairs whose progeny trait distributions are nondominated in terms of their mean and variance values (maximize both). Since simulated progeny are obtained through Monte Carlo simulation in advance of the growing season, identifying the nondominated parent pairs is a MOSO problem. To solve the mating design problem, Hunter and McClosky (2016) propose a two-step solution method that identifies these parent pairs in the first step.

We classify additional papers containing MOSO applications by application area in Table 1 . Whenever possible, we classify the papers according to their best fit with the application track descriptions at the 2017 Winter Simulation Conference (Chan et al. 2017). For each paper listed in Table 1, we also indicate what kind of decision variables make up the decision vector $\mathbf{x} \in \mathcal{X}$ and how many objectives the application contains. If the paper describes a general methodology followed by a case study, we indicate the number of objectives used in the case study. If the paper is an application-area review that includes MOSO concepts, we indicate that the paper is a review.

In Table 1, we adopt the problem taxonomy of Pasupathy and Henderson $(2006,2011)$ with minor changes. We say the feasible set $\mathcal{X}$ is (1) continuous if each decision variable can take any value in an interval subset of the real numbers, (2) integer-ordered if each decision variable can take on integer values, and (3) categorical if each decision variable can take on a finite number of noninteger values that may be categorical or unordered. Note that one can define a neighborhood structure on some categorical problems; once the neighborhood structure is defined, these problems may be 
Table 1. Example MOSO Applications Categorized by Area, the Number of Objectives in the Application or Case Study, and the Nature of the Feasible Set $\mathcal{X}$ (Categorical, Integer-Ordered, or Continuous)

\begin{tabular}{|c|c|c|}
\hline Area & Obj. & MOSO Application Papers \\
\hline \multirow[t]{3}{*}{ agriculture } & $d=2$ & categorical: plant breeding (Hunter and McClosky 2016) \\
\hline & $d=3$ & continuous: irrigation design (Crespo et al. 2010) \\
\hline & $d=4$ & categorical: land management under climate change (Klein et al. 2013) \\
\hline architecture & $d=2$ & categorical: earthmoving operations (Zhang 2008) \\
\hline \& construction & $d \geq 2$ & $\begin{array}{l}\text { review: building performance analysis, usually } d \in\{2,3\} \text { (Nguyen et al. } \\
\text { 2014) }\end{array}$ \\
\hline \multirow[t]{3}{*}{ aviation } & $d=2$ & integer-ordered: aircraft spare part management (Li et al. 2015a, 2015c) \\
\hline & & continuous: aircraft flight scheduling (Lee et al. 2007) \\
\hline & $d=3$ & integer-ordered: aircraft spare part management (Lee et al. 2008) \\
\hline \multirow[t]{3}{*}{ energy } & $d=2$ & $\begin{array}{l}\text { continuous: oil drilling (Kim 2014); design of burners in the combustion } \\
\text { chamber of a gas turbine (Büche et al. 2002) }\end{array}$ \\
\hline & $d=3$ & continuous: power plant design (Subramanyan et al. 2011) \\
\hline & $d \geq 2$ & $\begin{array}{l}\text { continuous: energy pricing in an electricity market as a stochastic } \\
\text { collaborative game between } d \text { players (Fliege and Xu 2011, p. 158) }\end{array}$ \\
\hline environment & $d=2$ & integer-ordered: groundwater remediation design (Singh and Minsker 2008) \\
\hline \& sustainability & $d \geq 2$ & $\begin{array}{l}\text { integer-ordered: dynamic flood control operation in a river-reservoir system } \\
\text { with up to } d=5 \text { (Prakash et al. 2015) }\end{array}$ \\
\hline \multirow[t]{4}{*}{ healthcare } & $d=2$ & $\begin{array}{l}\text { integer-ordered: capacity allocation in an emergency department or } \\
\text { obstetrics ward (Chen and Wang 2016; Lucidi et al. 2016); portable } \\
\text { ultrasound machine allocation (Huang 2016, p. 90) }\end{array}$ \\
\hline & & $\begin{array}{l}\text { continuous: patient flow between healthcare centers and hospital with } \\
d=8 \text { reduced to } d=2 \text { (Song et al. } 2016)\end{array}$ \\
\hline & $d=4$ & $\begin{array}{l}\text { integer-ordered: patient flow and capacity in a cancer treatment center } \\
\text { (Baesler and Sepulveda 2001); hospital inpatient flow process, solved as } \\
\text { three sets of paired objectives (Wang et al. 2015) }\end{array}$ \\
\hline & $d=5$ & continuous: cadaveric liver allocation policies (Feng et al. 2013) \\
\hline \multirow{2}{*}{$\begin{array}{l}\text { logistics, } \\
\text { supply chain, } \\
\& \text { transportation }\end{array}$} & $d=2$ & categorical: supply chain management (Ding et al. 2006) \\
\hline & & $\begin{array}{l}\text { integer-ordered: reduce congestion in a lighterage terminal (Zhou et al. } \\
\text { 2018); differentiated service inventory management (Chew et al. 2009); } \\
\text { supply chain management (Amodeo et al. 2009; Joines et al. 2002; Li et al } \\
\text { 2017); train traction system design (Dullinger et al. 2017) }\end{array}$ \\
\hline \multirow[t]{2}{*}{ manufacturing } & $d=2$ & integer-ordered: production line scheduling (Andersson et al. 2007) \\
\hline & $d \geq 2$ & continuous: injection molding (Villarreal-Marroquín et al. 2013) \\
\hline \multirow[t]{2}{*}{ military } & $d=2$ & continuous: fighter aircraft maintenance (Mattila and Virtanen 2014) \\
\hline & $d=3$ & continuous: military ground vehicle design for safety (Hoffenson et al. 2014) \\
\hline
\end{tabular}

mapped to a subset of an integer lattice and considered integer-ordered problems. For the purpose of Table 1, papers classified as categorical do not define such a neighborhood structure. Since some problems may contain a mix of variable types, we again adopt the convention of Pasupathy and Henderson (2011) that the feasible set $\mathcal{X}$ is categorical if any variables are categorical, integerordered if any variables are integer-ordered and none are categorical, and continuous otherwise.

Table 1 shows that MOSO problems already exist in areas as varied as agriculture, energy, environment and sustainability, healthcare, the military, and supply chain management, among others. Thus, the further development of efficient MOSO methods and algorithms is likely to have a significant impact on application areas and the ability to solve complex real-world problems. 


\subsection{Related Literature}

$\mathrm{MOO}$ and SOSO are relatively well-developed fields that provide a foundation for the development of MOSO theory, methods, and algorithms. In the sections that follow, we comment on the existing MOO and SOSO literature, define terms relevant to our problem and solution method taxonomy, and provide references to entry points for each area. We conclude the section with references to previous reviews on MOSO and remarks on our contribution.

1.2.1 Literature on Deterministic Multiobjective Optimization. Deterministic MOO methods usually consist of generating one or more efficient points. MOO has been an active research topic for over 100 years (see, e.g., Stadler 1979; Ehrgott 2012). To begin our discussion, we define several terms we use to classify MOO methods (and later, MOSO methods).

First, since only one solution can be implemented in practice, MOO solution methods usually involve generating the Pareto-optimal solution that is preferred by the decision maker (Miettinen 1999). However, the decision maker can articulate preferences at varying points in the decisionmaking process. Following Hwang and Masud (1979), Miettinen (1999, p. 63) uses a classification of MOO methods based on when the preferences of the decision maker are articulated: no-preference methods generate an efficient point without regard to the decision maker's preference; a posteriori methods first characterize the efficient or Pareto set in an optimization stage, then allow the decision maker to express a preference in a separate MCDM stage; a priori methods incorporate the decision maker's preferences before the optimization is conducted; and interactive methods periodically solicit information from the decision maker to guide the optimization process. Miettinen (1999) notes that these classes overlap, and some methods fit into more than one category.

Second, MOO solution methods may fall under the category of scalarization or nonscalarization methods. Scalarization is the primary solution method for MOO problems (Miettinen 1999). Scalarization converts the MOO problem into a parameterized single-objective problem that can be solved using well-established single-objective optimization methods. Under appropriate regularity conditions, solving the single-objective scalarized problem usually results in an efficient point. Varying the scalarization parameters and solving each resulting problem usually creates a set of efficient points and a characterization of the Pareto set in the objective function space. Many scalarizing functions exist, the simplest of which is arguably the linear weighted sum: each objective is multiplied by a weight, and the weighted objectives are added together to form a single objective. In this case, the weights are the scalarization parameters. Since one single-objective problem is solved for each scalarization parameter value, an efficient single-objective optimization "engine" is required (Marler and Arora 2004, p. 390). In contrast, nonscalarization methods find efficient points in a variety of other ways. For example, one could use gradient information to find a descent direction on all objectives until arriving at a point that satisfies first-order necessary conditions in the multiobjective context (see, e.g., Miettinen 1999, pp. 37-57). Descent methods include the steepest descent method of Fliege and Svaiter (2000), the Newton-like method of Fliege et al. (2009), and the multiple gradient descent algorithm (MGDA) of Désidéri (2012). Other approaches include non-Pareto optimality and set-oriented methods. Non-Pareto optimality methods replace the concept of Pareto optimality with suitable alternatives, such as lexicographic or max-ordering relations. Set-oriented methods attempt to capture the entire efficient set and iterate on sets instead of points. We refer the reader to Wiecek et al. (2016) for more information on both scalarization and nonscalarization methods.

Since the MOO literature is vast, we also refer the reader to Chankong and Haimes (1983), Ehrgott (2005), Ehrgott et al. (2016), Ehrgott and Wiecek (2005), Eichfelder (2008), Miettinen (1999), and Wiecek et al. (2016) for theory and solution methods, and to Chinchuluun and Pardalos (2007), Marler and Arora (2004), and White (1990) for additional MOO overviews and applications. 
1.2.2 Literature on Single-Objective Simulation Optimization. SOSO solution methods involve optimizing an unknown, nonlinear objective function that can only be observed with stochastic error. SOSO methodology has been an active research area for over 30 years. The primary focus has been on solving SOSO problems without stochastic constraints; methods for SOSO problems with stochastic constraints have been developed more recently. As a source of theory and "engines," the SOSO literature holds particular importance for the future development of MOSO methods.

As in Hong and Nelson (2009) and Pasupathy and Ghosh (2013), we categorize SOSO (and later, MOSO) solution methods according to the type of feasible set for which they were designed (see Section 1.1 for definitions of categorical, integer-ordered, and continuous feasible sets). Solution methods for categorical feasible sets and "small" finite feasible sets are called ranking and selection $(\mathrm{R} \& \mathrm{~S})$ methods. $\mathrm{R} \& \mathrm{~S}$ methods require obtaining simulation replications from every decision point in the finite feasible space and usually provide some form of probabilistic guarantee on the sampling efficiency of the procedure or on the optimality gap of the returned solution. SOSO solution methods for integer-ordered sets and continuous sets require the corresponding feasible set $\mathcal{X}$ in Problem $S$ to be integer-ordered or continuous, respectively. If a solution method can accommodate more than one type of feasible set or can accommodate mixed variables, we say the method is (1) an R\&S method if it can accommodate categorical feasible sets and (2) an integer-ordered SOSO method if it can accommodate integer-ordered feasible sets and cannot accommodate categorical feasible sets.

Many literature overviews and tutorials provide entry points to the SOSO literature. For the reader new to SOSO, we recommend Pasupathy and Ghosh (2013) as a companion to this article. Other overviews include Amaran et al. (2016), Fu (1994, 2002), Hong and Nelson (2009), and Jian and Henderson (2015), and the books by Chen et al. (2013), Chen and Lee (2010), and Fu (2015). Some subsets of the SOSO literature have their own overviews. For entry points to the R\&S literature, see Goldsman (2015), Hunter and Nelson (2017), and Kim and Nelson (2006). For SOSO methods on integer-ordered sets, including with stochastic constraints, see Nelson (2010). The literature on continuous SOSO methods consists primarily of (1) stochastic approximation (SA), a stochastic version of Newton's method, and (2) sample average approximation (SAA). For entry points to the SOSO literature on SA, see Kushner and Yin (2003), Pasupathy and Kim (2011), and Pasupathy and Ghosh (2013, pp. 138-145). For entry points to the SAA literature, including SAA with stochastic constraints, see Homem-de-Mello and Bayraksan (2015), Kim et al. (2015), Ruszczyński and Shapiro (2003), Shapiro (2003), and Shapiro et al. (2009).

1.2.3 Previous MOSO Surveys and Our Contribution. MOSO methods usually involve generating one or more efficient points when multiple unknown, nonlinear objective functions can only be observed with stochastic error. Compared to MOO and SOSO, the corresponding literature on MOSO is relatively small. However, some surveys do exist. Among the earliest surveys is Friedman (1987), which briefly discusses MOSO as part of a broader survey on the analysis of multivariate response simulations. Several surveys cover SOSO topics and have a section that also addresses MOSO topics, including Jacobson and Schruben (1989, pp. 2-3), Tekin and Sabuncuoglu (2004, p. 1075), Gutjahr (2011, pp. 62-63), Andradóttir (2015, pp. 287-288), Xu et al. (2015, p. 17), and Yoon and Bekker (2017, pp. 41-43). Abdelaziz (2012) and Gutjahr and Pichler (2016, published online in 2013) touch on MOSO topics, but their primary focus lies on problems with more structure than our Problem $S$, such as multiobjective stochastic programming. Few surveys are dedicated to MOSO under the general Problem $S$; these surveys include Evans et al. (1991) and Rosen et al. (2008).

Among these dedicated surveys, none focus on the optimization step of a posteriori MOSO methods, that is, methods that characterize the entire efficient set (henceforth, simply "a posteriori 
MOSO" methods; see Section 1.3). Rosen et al. (2008) state that at the time of writing, there were no MOSO methods specifically designed for a posteriori MOSO. They remark that the lack of literature may be due to the computational intensity of the problem. Indeed, the literature we cover has been published since 2008.

With the recent advances in available parallel computing power, we view this article as a timely beginning reference for researchers who would like to work in this area. Our aim is to lay the foundation for future work in provably convergent a posteriori MOSO methods by providing a single reference containing problem and solution formulations, metrics for evaluating algorithms, a survey of foundational results and state-of-the-art methods, and a discussion of open questions (see also Section 2.4).

\subsection{Terminology and Notation}

Whenever it is required for clarity, such as in our section on scope (Section 2.4), we refer to methods employed during the optimization step of a posteriori MOSO as a posteriori MOSO methods, and acknowledge here that we ignore the second MCDM step of selecting one decision point from the efficient set. Elsewhere in the article, we drop the specification "a posteriori" for this class of methods and refer to them simply as MOSO methods.

In the MOO literature, there is some flexibility regarding terms for the solution to Problem $S$ in the decision space and its image in the objective space (Ehrgott 2005, pp. 24, 60). For guidance in determining a convention for this article, we turn to Ehrgott (2012), who discusses the history of MOO. Specifically, Vilfredo Pareto's contribution to the concept of Pareto optimality was exclusively in the objective space. When Kuhn and Tucker (1951) discussed the concept of multiobjective optimization, they did not reference Pareto; instead, they cited Koopmans (1951) and used the term "efficient." To be consistent with Pareto's contributions and to maintain distinct terms for each space, henceforth, we adopt the convention that efficient points are in the decision space and Pareto-optimal points or Pareto points are in the objective function space.

We use the following notation in the remainder of the article. The set of all $d$-dimensional integer-valued vectors is $\mathbb{Z}^{d} \subset \mathbb{R}^{d}$. Usually, capital letters denote random variables $(X)$, script capital letters denote sets $(\mathcal{A})$, vectors appear in bold $(\mathbf{x})$, and random vectors appear in capital bold $(\boldsymbol{X})$. The $d$-dimensional vector of zeros is $\mathbf{0}_{d}$. If $\mathbf{g}: \mathcal{X} \subseteq \mathbb{R}^{q} \rightarrow \mathbb{R}^{d}$ is a vector-valued function, then for some set $\mathcal{S} \subseteq \mathcal{X}$, the set $\mathrm{g}(\mathcal{S})$ is the image of the set $\mathcal{S}, \mathrm{g}(\mathcal{S}):=\{\mathrm{g}(\mathrm{x}): \mathrm{x} \in \mathcal{S}\}$. The sum of two sets $\mathcal{A}$ and $\mathcal{B}$ is the Minkowski sum, $\mathcal{A}+\mathcal{B}:=\{\mathbf{a}+\mathbf{b}: \mathbf{a} \in \mathcal{A}, \mathbf{b} \in \mathcal{B}\}$. We say that a sequence of random variables $X_{1}, X_{2}, \ldots$ converges with probability 1 (w.p.1) to a random variable $X$ if for all $\varepsilon>0, \mathbb{P}\left\{\lim _{n \rightarrow \infty}\left|X_{n}-X\right|<\varepsilon\right\}=1$ (Casella and Berger 2002, p. 234). For a sequence of events $\left\{E_{n}\right\}$ defined in a probability space, we say $E_{n}$ infinitely often (i.o.) if infinitely many of $E_{n}$ occur, where $E_{n}$ i.o. $=\limsup _{n} E_{n}=\cap_{n=1}^{\infty} \cup_{j=n}^{\infty} E_{j}$ (Billingsley 1995, pp. 52-53). We require notions of distance. Let $\mathcal{A} \subset \mathbb{R}^{q}$ and $\mathcal{B} \subset \mathbb{R}^{q}$ be two nonempty, bounded sets. Then (1) $d\left(\mathbf{x}, \mathbf{x}^{\prime}\right)=\left\|\mathbf{x}-\mathbf{x}^{\prime}\right\|$ is the Euclidean distance between two points $\mathbf{x}, \mathbf{x}^{\prime} \in \mathbb{R}^{q} ;(2) d(\mathbf{x}, \mathcal{B})=\inf _{\mathbf{x}^{\prime} \in \mathcal{B}}\left\|\mathbf{x}-\mathbf{x}^{\prime}\right\|$ is the distance from the point $\mathbf{x} \in \mathbb{R}^{q}$ to the set $\mathcal{B} ;(3) \mathbb{D}(\mathcal{A}, \mathcal{B})=\sup _{x \in \mathcal{A}} d(x, \mathcal{B})$ is the distance from set $\mathcal{A}$ to set $\mathcal{B}$; and $(4) \mathbb{H}(\mathcal{A}, \mathcal{B}):=\max \{\mathbb{D}(\mathcal{A}, \mathcal{B}), \mathbb{D}(\mathcal{B}, \mathcal{A})\}$ is the Hausdorff distance between sets $\mathcal{A}$ and $\mathcal{B}$.

\section{THE MULTIOBJECTIVE SIMULATION OPTIMIZATION PROBLEM SETTING}

To begin our in-depth discussion of MOSO, we first define optimality concepts. Then, we write a formal problem statement, discuss solution quality metrics, and comment on our scope. Henceforth, we focus on the version of Problem $S$ without stochastic constraints:

$$
\text { Problem } M: \quad \operatorname{minimize}_{\mathbf{x} \in X} \quad\left\{\mathbf{g}(\mathbf{x})=\left(g_{1}(\mathbf{x}), \ldots, g_{d}(\mathbf{x})\right):=\left(\mathbb{E}\left[G_{1}(\mathbf{x}, \xi)\right], \ldots, \mathbb{E}\left[G_{d}(\mathbf{x}, \xi)\right]\right)\right\},
$$


where $\mathrm{g}: \mathcal{X} \rightarrow \mathbb{R}^{d}$ is a vector-valued function. With the exception of work on SAA cited in Section 4, at the time of writing, we know of no other MOSO methods in the scope of our article (see Section 2.4) that are designed specifically to handle stochastic constraints.

\subsection{Optimality Concepts}

In this section, we define what we mean by global and local solutions to Problem $M$. Unless otherwise cited, the majority of the content in this section can be found in standard MOO textbooks, such as Ehrgott (2005) and Miettinen (1999). The concepts we discuss are illustrated in Figure 1 for a simple case of a problem with two objectives and a continuous feasible set.

2.1.1 Global Optimality. To define concepts of global optimality in Problem $M$, first, we define the concept of dominance in the objective function space.

Definition 2.1. Let $\mathbf{x}_{1}, \mathbf{x}_{2} \in \mathcal{X}$ and $d \geq 2$. For vectors $\mathbf{g}\left(\mathbf{x}_{1}\right)$ and $\mathbf{g}\left(\mathbf{x}_{2}\right)$, we say that

(1) $\mathbf{g}\left(\mathbf{x}_{1}\right)$ weakly dominates $\mathbf{g}\left(\mathbf{x}_{2}\right)$, written as $\mathbf{g}\left(\mathbf{x}_{1}\right) \leqq \mathbf{g}\left(\mathbf{x}_{2}\right)$, if $g_{k}\left(\mathbf{x}_{1}\right) \leq g_{k}\left(\mathbf{x}_{2}\right)$ for all $k=$ $1, \ldots, d$

(2) $\mathbf{g}\left(\mathbf{x}_{1}\right)$ dominates $\mathbf{g}\left(\mathbf{x}_{2}\right)$, written as $\mathbf{g}\left(\mathbf{x}_{1}\right) \leq \mathbf{g}\left(\mathbf{x}_{2}\right)$, if $\mathbf{g}\left(\mathbf{x}_{1}\right) \leqq \mathbf{g}\left(\mathbf{x}_{2}\right)$ and $\mathbf{g}\left(\mathbf{x}_{1}\right) \neq \mathbf{g}\left(\mathbf{x}_{2}\right)$; and

(3) $\mathbf{g}\left(\mathbf{x}_{1}\right)$ strictly dominates $\mathbf{g}\left(\mathbf{x}_{2}\right)$, written as $\mathbf{g}\left(\mathbf{x}_{1}\right)<\mathbf{g}\left(\mathbf{x}_{2}\right)$, if $g_{k}\left(\mathbf{x}_{1}\right)<g_{k}\left(\mathbf{x}_{2}\right)$ for all $k=$ $1, \ldots, d$.

Using the concept of dominance, we now define efficient and weakly efficient points.

Definition 2.2. A decision point $\mathbf{x}^{*} \in \mathcal{X}$ is

(1) an efficient point if there does not exist another point $\mathbf{x} \in \mathcal{X}$ such that $\mathbf{g}(\mathbf{x}) \leq \mathbf{g}\left(\mathbf{x}^{*}\right)$ and

(2) a weakly efficient point if there does not exist another point $\mathbf{x} \in \mathcal{X}$ such that $\mathbf{g}(\mathbf{x})<\mathbf{g}\left(\mathbf{x}^{*}\right)$.

Notice that all efficient points are also weakly efficient points. We define a Pareto point and a weakly Pareto point as the image of an efficient point and a weakly efficient point, respectively.

In the presence of conflicting objectives, there may exist more than one efficient point or weakly efficient point, which we collect into sets, as follows.

Definition 2.3. We define the following sets:

(1) The efficient set, $\mathcal{E} \subseteq \mathcal{X}$, is the set of all efficient points.

(2) The weakly efficient set, $\mathcal{E}^{\mathrm{w}}$, where $\mathcal{E} \subseteq \mathcal{E}^{\mathrm{w}} \subseteq \mathcal{X}$, is the set of all weakly efficient points.

The Pareto set is the image of the efficient set $\mathcal{P}:=\mathrm{g}(\mathcal{E})$, and the weakly Pareto set is the image of the weakly efficient set $\mathcal{P}^{\mathrm{w}}:=\mathrm{g}\left(\mathcal{E}^{\mathrm{w}}\right)$, where $\mathcal{P} \subseteq \mathcal{P}^{\mathrm{w}}$.

Finally, we define two points in the objective space that may be used to bound the Pareto set $\mathcal{P}$, called the ideal and nadir points. The ideal point is constructed by minimizing each objective function and storing the information as $\mathbf{g}^{\text {ideal }}:=\left(\min _{\mathbf{x} \in \mathcal{X}} g_{1}(\mathbf{x}), \min _{\mathbf{x} \in \mathcal{X}} g_{2}(\mathbf{x}), \ldots, \min _{\mathbf{x} \in \mathcal{X}} g_{d}(\mathbf{x})\right)=$ $\left(\min _{\mathbf{x} \in \mathcal{E}} g_{1}(\mathbf{x}), \min _{\mathbf{x} \in \mathcal{E}} g_{2}(\mathbf{x}), \ldots, \min _{\mathbf{x} \in \mathcal{E}} g_{d}(\mathbf{x})\right)$, where the last equality assumes $\mathcal{E}$ is nonempty. Notice that the ideal point can only be the solution to Problem $M$ if none of the objectives conflict. Assuming the efficient set $\mathcal{E}$ is nonempty, the nadir point provides an upper bound on the Pareto set and is defined as $\mathbf{g}^{\text {nadir }}:=\left(\max _{\mathbf{x} \in \mathcal{E}} g_{1}(\mathbf{x}), \max _{\mathbf{x} \in \mathcal{E}} g_{2}(\mathbf{x}), \ldots, \max _{\mathbf{x} \in \mathcal{E}} g_{d}(\mathbf{x})\right)$. In two dimensions, one can obtain the nadir point from values used to create the ideal point (Ehrgott and Tenfelde-Podehl 2003). To see this, let $\mathbf{x}_{k}^{\min }=\operatorname{argmin} g_{k}(\mathbf{x})$ for all $k \in\{1,2\}$. Then $\mathbf{g}^{\text {nadir }}=$ $\left(g_{1}\left(\mathbf{x}_{2}^{\min }\right), g_{2}\left(\mathbf{x}_{1}^{\min }\right)\right)$. In more than two dimensions, the value of the nadir point may be more difficult to determine; see, e.g., Ehrgott and Tenfelde-Podehl (2003) and Kirlik and Sayin (2013). Some MOSO methods require the specification of a reference point; frequently, the reference point either 


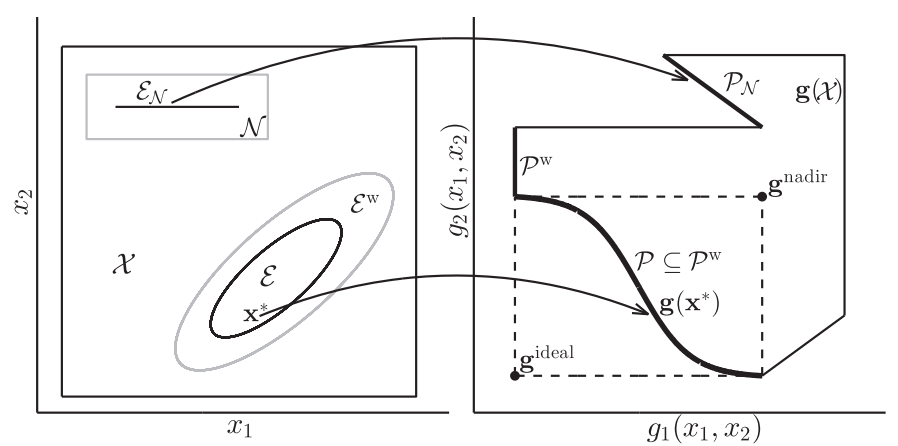

Fig. 1. For a biobjective example Problem $M$, the figure shows the feasible space $\mathcal{X}$ on the left and the objective function space $\mathrm{g}(\mathcal{X})$ on the right. The symbols in the figure are defined in Section 2.1.

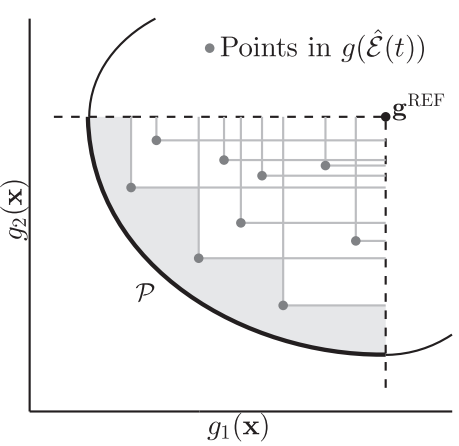

Fig. 2. The area of the shaded region equals $\mathbb{H} \mathbb{V} \mathbb{D}\left(\mathcal{P}, \mathbf{g}(\hat{\mathcal{E}}(t)), \mathrm{g}^{\mathrm{REF}}\right)$, defined in Section 2.3.2.

dominates the Pareto set, such as $\mathrm{g}^{\text {ideal }}$, or is dominated by points in the Pareto set, such as $\mathrm{g}^{\text {nadir }}$. We refer to "dominating" reference points as $\mathrm{g}^{\text {ref }}$ and to "dominated" reference points as $\mathrm{g}^{\mathrm{REF}}$.

2.1.2 Local Optimality. If a neighborhood structure can be defined for the feasible points (e.g., $X \subseteq \mathbb{R}^{q}$ or $X \subseteq \mathbb{Z}^{q}$ ), then there may exist local solutions to Problem $M$. That is, there may exist points that are efficient in a neighborhood but that are not necessarily globally efficient. Given such a neighborhood defined in the decision space, we now define local optimality concepts.

Definition 2.4. Let $\mathbf{x}^{*} \in \mathcal{X}$. Given an appropriate neighborhood $\mathcal{N}\left(\mathbf{x}^{*}\right)$, we say $\mathbf{x}^{*}$ is

(1) a local efficient point on $\mathcal{N}$ if there does not exist $\mathbf{x} \in \mathcal{N}\left(\mathbf{x}^{*}\right) \cap \mathcal{X}$ such that $\mathbf{g}(\mathbf{x}) \leq \mathbf{g}\left(\mathbf{x}^{*}\right)$, and

(2) a local weakly efficient point on $\mathcal{N}$ if there does not exist $\mathrm{x} \in \mathcal{N}\left(\mathrm{x}^{*}\right) \cap \mathcal{X}$ such that $\mathrm{g}(\mathrm{x})<\mathrm{g}\left(\mathrm{x}^{*}\right)$.

We also collect the local efficient and local weakly efficient points into local efficient and local weakly efficient sets. To define these concepts formally, we require definitions for the neighborhood of a set and the deleted neighborhood of a set. Given the previous notion of an appropriate neighborhood of a point $\mathcal{N}(\mathrm{x})$, we define the neighborhood of a set $\mathcal{S}$ as $\mathcal{N}(\mathcal{S}):=\cup_{\mathrm{x} \in \mathcal{S}} \mathcal{N}(\mathrm{x})$. The deleted neighborhood of a set $\mathcal{S}$ is $\mathcal{N}^{\prime}(\mathcal{S}):=\mathcal{N}(\mathcal{S}) \backslash \mathcal{S}$. Then we have the following definitions (see Cooper et al. 2017, 2018; Deb 1999; Kim and Ryu 2011a; Li et al. 2015a).

Definition 2.5. Given an appropriate neighborhood $\mathcal{N}(\mathbf{x})$ for each $\mathbf{x} \in \mathcal{X}$ :

(1) A set $\mathcal{E}_{\mathcal{N}}$ is a local efficient set on $\mathcal{N}$ if (a) for each $\mathrm{x}^{*} \in \mathcal{E}_{\mathcal{N}}, \mathrm{x}^{*}$ is a local efficient point on $\mathcal{N}\left(\mathbf{x}^{*}\right)$; (b) no points in $\mathbf{g}\left(\mathcal{E}_{\mathcal{N}}\right)$ dominate other points in $\mathrm{g}\left(\mathcal{E}_{\mathcal{N}}\right)$; and (c) for each $\mathbf{x} \in$ $\mathcal{N}^{\prime}\left(\mathcal{E}_{\mathcal{N}}\right) \cap \mathcal{X}$, there exists $\mathrm{x}^{*} \in \mathcal{E}_{\mathcal{N}}$ such that $\mathrm{g}\left(\mathrm{x}^{*}\right) \leq \mathrm{g}(\mathrm{x})$.

(2) A set $\mathcal{E}_{\mathcal{N}}^{\mathrm{w}}$ is a local weakly efficient set on $\mathcal{N}$ if (a) for each $\mathbf{x}^{*} \in \mathcal{E}_{\mathcal{N}}^{\mathrm{w}}$, $\mathbf{x}^{*}$ is a local weakly efficient point on $\mathcal{N}\left(\mathbf{x}^{*}\right)$; (b) no points in $\mathrm{g}\left(\mathcal{E}_{\mathcal{N}}^{\mathrm{w}}\right)$ strictly dominate other points in $\mathrm{g}\left(\mathcal{E}_{\mathcal{N}}^{\mathrm{w}}\right)$; and (c) for each $\mathbf{x} \in \mathcal{N}^{\prime}\left(\mathcal{E}_{\mathcal{N}}^{\mathrm{w}}\right) \cap \mathcal{X}$, there exists $\mathbf{x}^{*} \in \mathcal{E}_{\mathcal{N}}^{\mathrm{w}}$ such that $\mathbf{g}\left(\mathbf{x}^{*}\right) \leqq \mathrm{g}(\mathbf{x})$.

Thus, each point in a local efficient set must be a local efficient point on $\mathcal{N}$, the image of each point in the set should not be dominated by the images of any other points in the set, and each point in the deleted neighborhood of the local efficient set must be dominated by a point in the local efficient set. Further, the local Pareto set is $\mathcal{P}_{\mathcal{N}}:=\mathrm{g}\left(\mathcal{E}_{\mathcal{N}}\right)$ and the local weakly Pareto set is $\mathcal{P}_{\mathcal{N}}^{\mathrm{w}}:=\mathrm{g}\left(\mathcal{E}_{\mathcal{N}}^{\mathrm{w}}\right)$. 
2.1.3 Convexity. If the feasible set $\mathcal{X}$ is continuous and convex, and if all objective functions $g_{k}(\mathbf{x}), k=1, \ldots, d$ are convex, then Problem $M$ is convex (Miettinen 1999, p. 7). If Problem $M$ is convex, then all local efficient points are also (global) efficient points (Miettinen 1999, p. 12, Theorem 2.2.3). Further, the objective function space has a special structure: the $\operatorname{set} \mathbf{g}(\mathcal{X})+\left\{\mathbf{y} \in \mathbb{R}^{d}\right.$ : $\left.\mathbf{0}_{d} \leqq \mathbf{y}\right\}$ is a convex set (see Section 1.3 for notation; Wiecek et al. 2016, p. 747). To understand this statement, notice that $\left\{\mathrm{y} \in \mathbb{R}^{d}: \mathbf{0}_{d} \leqq \mathrm{y}\right\}$ is the nonnegative orthant, so that for each $\mathbf{x} \in \mathcal{X}$, the set $\mathbf{g}(\mathbf{x})+\left\{\mathbf{y} \in \mathbb{R}^{d}: \mathbf{0}_{d} \leqq \mathbf{y}\right\}$ contains $\mathbf{g}(\mathbf{x})$ and the points it dominates. Then the fact that the set $\mathrm{g}(\mathcal{X})+\left\{\mathrm{y} \in \mathbb{R}^{d}: \mathbf{0}_{d} \leqq \mathrm{y}\right\}$ is convex implies the existence of a supporting hyperplane for any point in the Pareto set $\mathcal{P}$. Notice that the right side of Figure 1 does not depict a convex version of Problem $M$, while Figure 2 does. If known in advance, the properties of convexity can be exploited for MOSO algorithmic efficiency.

\subsection{Problem Statement}

Since the objective functions in Problem $M$ are unknown, we assume the existence of an oracle capable of producing consistent estimators of all unknown functions for each $\mathbf{x} \in \mathcal{X}$. That is, we assume the oracle produces estimators $\hat{G}_{k}(\mathbf{x}, n)$, where $\hat{G}_{k}(\mathbf{x}, n) \rightarrow g_{k}(\mathbf{x})$ w.p.1 as the simulation effort $n \rightarrow \infty$ for each $k=1, \ldots, d$ and $\mathbf{x} \in \mathcal{X}$; let $\hat{G}(\mathbf{x}, n):=\left(\hat{G}_{1}(\mathbf{x}, n), \ldots, \hat{G}_{d}(\mathbf{x}, n)\right)$. Then a general, and perhaps ambitious, version of the MOSO problem statement is, given an oracle that produces the estimator $\hat{G}(\mathbf{x}, n)$ of $\mathbf{g}(\mathbf{x})$ for each $\mathbf{x} \in \mathcal{X}$, find the solution to Problem $M$, which is the efficient set $\mathcal{E}$. In what follows, depending on the feasible set $\mathcal{X}$, we may consider less ambitious versions of this problem statement. For example, if $\mathcal{X}$ is integer-ordered or continuous, we instead consider algorithms that find a local efficient set $\mathcal{E}_{\mathcal{N}}$ as the solution to Problem $M$, given an appropriate neighborhood $\mathcal{N}$. We further discuss the scope of this article in Section 2.4.

\subsection{Algorithmic Efficiency and Solution Quality Metrics}

Given our problem statement, how do we assess the efficiency of a MOSO solution method? Analogous to the SOSO metrics described in Pasupathy and Ghosh (2013, p. 124), we measure the efficiency of MOSO solution methods using solution quality metrics that are based on the true objective vectors of the estimated solution, $\mathbf{g}(\hat{\mathcal{E}}(t))$, as a function of the total simulation work done, $t$. Here, the estimated solution $\hat{\mathcal{E}}(t)$ is a set constructed from the estimators $\{\hat{G}(X, N(X))\}$, where each $X \in X$ is a feasible point visited by the MOSO algorithm, $N(X)$ is the number of simulation replications spent at the visited point $X$, and both quantities may be random. The cardinality of $\hat{\mathcal{E}}(t)$ may be finite or infinite.

In this section, we consider solution quality metrics from the point of view of comparing algorithms on a test problem for which all function values and the true solution are known, and hence quantities such as $\mathbf{g}(\hat{\mathcal{E}}(t))$ can be calculated exactly as a function of $t$. We formulate two solution quality metrics for the MOSO context: coverage error and hypervolume difference. Many other MOO metrics could be adapted for MOSO; see Audet et al. (2018), Faulkenberg and Wiecek (2010), Jiang et al. (2014), Vanderpooten et al. (2017), Wu and Azarm (2001), and Zitzler et al. (2003).

2.3.1 Coverage Error. Based on Sayin (2000) and Eichfelder (2008, pp. 101-107), one can seek a solution set $\hat{\mathcal{E}}(t)$ that "covers" the true efficient set, in the sense that all portions of the Pareto set are well represented. Using definitions directly analogous to those in Sayin (2000), one could define coverage error using the random variable $\mathbb{D}(\mathcal{P}, \mathbf{g}(\hat{\mathcal{E}}(t)))$, which is the distance from the Pareto set $\mathcal{P}$ to $\mathrm{g}(\hat{\mathcal{E}}(t))$, as a function of $t$. However, unlike in the MOO context, there may exist points in $\mathbf{g}(\hat{\mathcal{E}}(t))$ that are dominated by other points in $\mathbf{g}(\hat{\mathcal{E}}(t))$. Since $\mathbb{D}(\mathcal{P}, \mathbf{g}(\hat{\mathcal{E}}(t)))=0$ when $\mathcal{P} \subseteq \mathrm{g}(\hat{\mathcal{E}}(t)))$, this metric does not penalize returning "extra" dominated points. Thus, for 
a MOSO context, as in Cooper et al. $(2017,2018)$, we propose using Hausdorff distance as a metric for coverage error. We define the coverage error of the estimator $\hat{\mathcal{E}}(t)$ as the random variable $\mathbb{H}(\mathcal{P}, \mathbf{g}(\hat{\mathcal{E}}(t)))$, and we define the local coverage error of the estimator $\hat{\mathcal{E}}(t)$ as the random variable $\min \mathcal{P}_{\mathcal{N}} \mathbb{H}\left(\mathcal{P}_{\mathcal{N}}, \mathbf{g}(\hat{\mathcal{E}}(t))\right)$, where the minimum is taken over all possible local Pareto sets $\mathcal{P}_{\mathcal{N}}$ for each value of $t$. (Notice that the particular $\mathcal{P}_{\mathcal{N}}$ for which the minimum is achieved may be a function of $t$.) Thus, the quality of the solution $\hat{\mathcal{E}}(t)$ can be measured using quantiles of the coverage error or local coverage error, or using the expected coverage error, $\mathbb{E}[\mathbb{H}(\mathcal{P}, \mathrm{g}(\hat{\mathcal{E}}(t)))]$, or expected local coverage error, $\mathbb{E}\left[\min _{\mathcal{P}_{\mathcal{N}}} \mathbb{H}\left(\mathcal{P}_{\mathcal{N}}, \mathrm{g}(\hat{\mathcal{E}}(t))\right)\right]$, as a function of $t$.

2.3.2 Hypervolume Difference. A metric called the hypervolume difference may also be used to evaluate MOSO solution methods (see, e.g., Branke et al. 2016; Faulkenberg and Wiecek 2010, p. 428). To define the hypervolume difference, first, we define the hypervolume of a set, which is also called the S-metric (Zitzler 1999, pp. 43-45). Loosely speaking, the hypervolume of a set $\mathcal{A} \subset \mathbb{R}^{d}$ with respect to a (dominated) reference point $\mathrm{g}^{\mathrm{REF}}$ is the volume of the set of points that at least one point in $\mathcal{A}$ dominates and that $\mathrm{g}^{\mathrm{REF}}$ is dominated by; call this set $\mathcal{G}\left(\mathcal{A}, \mathrm{g}^{\mathrm{REF}}\right):=\cup_{\mathrm{g}(\mathrm{x}) \in \mathcal{A}}\left\{\mathrm{y} \in \mathbb{R}^{d}: \mathrm{g}(\mathbf{x}) \leq \mathrm{y} \leq \mathrm{g}^{\mathrm{REF}}\right\}$. Then the hypervolume of $\mathcal{A}$ is $\mathbb{H} \mathbb{V}\left(\mathcal{A}, \mathrm{g}^{\mathrm{REF}}\right):=\Lambda\left(\mathcal{G}\left(\mathcal{A}, \mathrm{g}^{\mathrm{REF}}\right)\right)$, where $\Lambda(\cdot)$ denotes the Lebesgue measure. Now define the hypervolume difference of the sets $\mathcal{A}, \mathcal{B} \subset \mathbb{R}^{d}$ as $\mathbb{H} \mathbb{V} \mathbb{D}\left(\mathcal{A}, \mathcal{B}, \mathrm{g}^{\mathrm{REF}}\right):=\Lambda\left(\mathcal{G}\left(\mathcal{A}, \mathrm{g}^{\mathrm{REF}}\right)\right)+\Lambda\left(\mathcal{G}\left(\mathcal{B}, \mathrm{g}^{\mathrm{REF}}\right)\right)-$ $2 \Lambda\left(\mathcal{G}\left(\mathcal{A}, \mathbf{g}^{\mathrm{REF}}\right) \cap \mathcal{G}\left(\mathcal{B}, \mathbf{g}^{\mathrm{REF}}\right)\right)$.

In the special case of the hypervolume difference between the Pareto set and the true objective values of the estimated efficient set, we have that the hypervolume difference equals the random variable $\mathbb{H} \mathbb{V} \mathbb{D}\left(\mathcal{P}, \mathrm{g}(\hat{\mathcal{E}}(t)), \mathrm{g}^{\mathrm{REF}}\right)=\mathbb{H} \mathbb{V}\left(\mathcal{P}, \mathrm{g}^{\mathrm{REF}}\right)-\mathbb{H} \mathbb{V}\left(\mathrm{g}\left(\hat{\mathcal{E}}(t), \mathrm{g}^{\mathrm{REF}}\right)\right.$ as a function of $t$. This result follows because for every point in $\mathrm{g}(\hat{\mathcal{E}}(t))$, there exists a point in $\mathcal{P}$ that weakly dominates it (see Figure 2). To evaluate the quality of the solution $\mathbf{g}(\hat{\mathcal{E}}(t))$ with respect to the "nearest" local Pareto set $\mathcal{P}_{\mathcal{N}}$, one may calculate the local hypervolume difference as the random variable $\min \mathcal{P}_{\mathcal{N}}^{*} \mathbb{H} \mathbb{V} \mathbb{D}\left(\mathcal{P}_{\mathcal{N}}^{*}, \mathbf{g}(\hat{\mathcal{E}}(t)), \mathbf{g}^{\mathrm{REF}}\right)$, where the minimum is taken over all possible local Pareto sets $\mathcal{P}_{\mathcal{N}}^{*}$ such that for every point in $\mathrm{g}(\hat{\mathcal{E}}(t))$ there exists a point in $\mathcal{P}_{\mathcal{N}}^{*}$ that dominates it. The solution quality of $\mathbf{g}(\hat{\mathcal{E}}(t))$ can be measured as quantiles of the hypervolume difference or local hypervolume difference, or using the expected hypervolume difference, $\mathbb{E}\left[\mathbb{H} \mathbb{V} \mathbb{D}\left(\mathcal{P}, \mathbf{g}(\hat{\mathcal{E}}(t)), \mathrm{g}^{\mathrm{REF}}\right)\right]$, or the expected local hypervolume difference, $\mathbb{E}\left[\min \mathcal{P}_{N}^{*} \mathbb{H} \mathbb{V} \mathbb{D}\left(\mathcal{P}_{\mathcal{N}}^{*}, \mathrm{~g}(\hat{\mathcal{E}}(t)), \mathrm{g}^{\mathrm{REF}}\right)\right]$, as a function of $t$.

Calculating the hypervolume of a set may be a computationally intensive task. However, numerous methods exist for calculating the hypervolume of a finite set, due to the popularity of hypervolume-based metrics in the MOO context and its relationship to Klee's measure problem (Chan 2013; Yildiz and Suri 2012). We refer the reader to, e.g., Lacour et al. (2017) and references therein for more on calculating the hypervolume of a finite set.

\subsection{Scope}

Given the extensive MOO literature, there are many possible approaches to solving the MOSO problem. Some approaches may result in disagreement, as noted by Rosenthal (1985, p. 138):

Clearly, ... a rational person would never deliberately select a dominated point. This is probably the only important statement in multiobjective optimization that can be made without the possibility of generating some disagreement.

In the sections that follow, we discuss the scope of this article in detail (see Section 1.3 for terminology). Specifically, we consider a posteriori multiobjective ranking and selection (MORS) methods for finite $\mathcal{X}$, and a posteriori MOSO methods and algorithms that provably converge to a local efficient set under the natural ordering for integer-ordered and continuous $\mathcal{X}$. We emphasize 
results and methods that represent, in our opinion, those that are the most fundamental or well developed, and that serve as a starting point for future work addressing the problem statement in Section 2.2. When space does not allow detailed coverage, we provide references for the interested reader.

2.4.1 Articulation of the Decision Maker's Preferences. Consistent with our problem statement, we cover only a posteriori MOSO methods, that is, methods that generate an entire local or global efficient set and that allow the decision maker to express a preference after the optimization is conducted, instead of before or during. We adopt the philosophy of Schmeiser (2008), which we extend to the a posteriori MOSO context as follows. Schmeiser (2008) states that SOSO solution methods should require the decision maker to provide only the problem context and the desired solution quality. For a posteriori MOSO, providing the problem context means providing the simulation oracle and determining the objectives. Specifying the desired solution quality means specifying some measure of the quality of the estimated efficient set or specifying a total simulation budget $t$ that limits the number of simulation oracle calls. As such, we do not consider interactive methods that repeatedly solicit information from the decision maker, even if the goal is to characterize some desirable subset of the efficient set. For the interested reader, we provide several references to the literature on no-preference, a priori, and interactive MOSO methods below. Since a priori methods are arguably the most well developed, we provide the most detail in that section.

No-Preference MOSO Methods. No-preference MOSO methods return a single point in the efficient set without regard to the decision maker's preference. Any scalarization method with arbitrarily chosen parameters that provably converges to an efficient point could be considered a no-preference method. While no-preference methods could be used together with, e.g., random restarts to generate multiple points in the efficient set, our interest lies in methods specifically designed to converge to a local efficient set. When drawing the line between no-preference and a posteriori MOSO methods for this article, we look primarily at the authors' contributions with regard to retrieving an entire local efficient set. For example, we cover Fliege and Xu (2011) in Section 4.3.1 because the authors develop a new scalarization with properties pertinent to retrieving an entire efficient set, even though their numerical implementation uses equidistant or randomly chosen weights in the scalarization. Poirion et al. (2017) develop a stochastic version of MGDA (Désidéri 2012) that is implemented with random restarts, but their main contributions surround developing and proving the convergence of an algorithm that finds a single efficient point without regard to the decision maker's preference. Since there is no contribution specific to finding the entire efficient set, we classify it as a no-preference method. For more on no-preference MOSO methods, we refer the reader to Rosen et al. (2008, pp. 335-336).

A Priori MOSO Methods. A priori MOSO methods require the decision maker to express preferences before the optimization is conducted. Such preferences can be expressed by specifying, e.g., scalarization parameters or a utility function. Since the preferences are specified before the optimization process, usually only a few estimated efficient points are returned to the user. We refer the reader to the discussion in Rosen et al. (2008, pp. 332-334) and the following notable or more recent a priori MOSO methods: (1) Dudewicz and Taneja $(1978,1981)$ discuss two-stage MORS procedures for selecting the best among several $d$-variate normal populations, where the best population is defined through a user-defined preference function; (2) Butler et al. (2001), Frazier and Kazachkov (2011), and Merrick et al. (2015) discuss solving the MORS problem with utility functions; (3) Mattila and Virtanen (2015) develop MOCBA-p for MORS, which is a variant of the Multiobjective Optimal Computing Budget Allocation (MOCBA) (Lee et al. 2010) (see Section 3.1.1) that uses incomplete a priori preference information to find a subset of the efficient set; (4) Rosen 
et al. (2007) develop MOSO methods based on a preference model and response surface methodology; (5) Bonnel and Collonge (2014) provide general SAA results for optimizing a prespecified function over the weakly efficient set of a continuous MOSO problem; (6) Bonnel and Collonge (2015) present an SAA-based algorithm that minimizes a user-defined function over the Pareto set of a convex continuous MOSO problem with $d=2$ objectives; and (7) Mahdavi et al. (2013) reformulate the MOSO problem into a stochastically constrained problem for prespecified bounds on the constrained objectives. We remark here that all stochastically constrained SOSO methods could be considered a priori MOSO methods, since the constraint values must be specified in advance.

Interactive MOSO Methods. Interactive methods repeatedly solicit preference information from the decision maker to guide the optimization process. Interactive methods may be useful when simulation runs are expensive and the decision maker is readily available to provide input. We point the interested reader to the overviews by Tekin and Sabuncuoglu (2004, p. 1075) and Rosen et al. (2008, pp. 329-332); more recent methods include Gören et al. (2017) and Steponavičè et al. (2014).

2.4.2 Finding a Global Solution versus Finding a Local Solution. Apart from MORS methods, in which simulation replications are usually obtained from every point in the finite feasible set $X$ and the estimated solution is the global estimated best, MOSO methods can be divided into those that seek a local efficient set and those that seek the global efficient set. Among methods that seek the global efficient set, metaheuristics are especially popular. For example, see Amodeo et al. (2009), Baesler and Sepulveda (2000), Bianchi et al. (2009), Chen and Wang (2016), Chica et al. (2017), Ding et al. (2006), Eskandari and Geiger (2009), Gutjahr (2005, 2011), Jin and Branke (2005), Joines et al. (2002), Kuriger and Grant (2011), Lee et al. (2009), Lin et al. (2013), Siegmund et al. (2016), and Zhou et al. (2011) for heuristic and metaheuristic MOSO methods and applications.

Despite the popularity of metaheuristics for MOSO (and for MOO and SOSO, as noted by Brockhoff (2011), Coello Coello et al. (2007), and Deb (2009) and by Fu (2002), Hong and Nelson (2009), Hong et al. (2015), Nelson (2010), and Ólafsson (2006), respectively), our goal is to facilitate the advancement of literature on provably convergent MOSO algorithms. Fu (2002) provides a detailed discussion of why specialized simulation optimization algorithms should be developed and used for SOSO problems, and the same arguments apply to the MOSO context. In summary, such algorithms are efficient because they appropriately control stochastic error. Recent efforts on the part of the academic SOSO community, including various academic-industry partnerships with simulation software providers and the solver library at simopt.org (Henderson and Pasupathy 2018), are attempting to increase the mainstream use of state-of-the-art, efficient, and provably convergent SOSO algorithms (Nelson 2010) and, as they are further developed, MOSO algorithms.

Certainly, not all a posteriori MOSO methods that seek a global solution are metaheuristics. We refer the reader to Andradóttir (2015, pp. 287-288) for a recent discussion of random search methods for MOSO, including simulated annealing (e.g., Alrefaei and Diabat 2009), and to Zhang et al. (2017) and references therein for a stochastic kriging approach to estimating the Pareto front. Further, Multi-Objective Probabilistic Branch and Bound (MOPBnB) (Huang and Zabinsky 2014) is a MOSO method for integer-ordered feasible sets $\mathcal{X}$ that can also handle continuous variables and provably converges to the global efficient set. However, we adopt the philosophy that absent the assumption of global structure, methods that preclude the use of local solvers-especially those that exploit gradient or pseudo-gradient information-are likely to be inefficient. Given the infancy of MOSO methods in general, we focus on the discussion and development of efficient and provably convergent MOSO algorithms that seek a local solution for integer-ordered and continuous feasible sets. Global search methods that do not preclude the use of solvers that converge to a local efficient set can build upon these methods in the future. Thus, we omit the further discussion of 
metaheuristics and other algorithms that contain a global search mechanism on integer-ordered and continuous feasible spaces.

2.4.3 Natural versus Other Orderings. The MOO literature exists as a part of the broader vector optimization literature, which considers vector orderings other than the so-called natural ordering in Definition 2.1. See Miettinen (1999, pp. 23-25) for a brief introduction to the vector optimization literature, or see Jahn (2011) for a more thorough treatment. We consider only the natural ordering.

2.4.4 Other Related Literature Not Covered. We do not cover literature on (1) robust MOSO (Fliege and Werner 2014; Liu et al. 2017); (2) the multiobjective multiarmed bandit problem, which is related to the MORS problem (Drugan and Manderick 2015; Reverdy 2016; Yahyaa and Drugan 2015; Yahyaa et al. 2014a, 2014b, 2014c, 2015); or (3) multiobjective optimization for multifidelity simulation models with biased, and not noisy, objective vector estimators (Li et al. 2015b).

\section{MULTIOBJECTIVE RANKING AND SELECTION}

We now consider methods to solve Problem $M$ when the cardinality of the feasible region, $|X|$, is finite; the decision variables may be categorical; and the feasible points are known as systems. In particular, we require the number of systems to be small enough that we are able to sample from every system. Recall from the beginning of Section 2.4 that these problems are called MORS problems.

MORS problems and solution methods are somewhat unique in the MOSO literature. For MORS problems, the returned solution always estimates the global efficient set, and the solution method in the corresponding deterministic context is complete enumeration. Further, MORS methods can be used in a variety of ways beyond solving problems with categorical variables. MORS methods can be used for sample allocation within other algorithms (e.g., Chew et al. 2009; Lee et al. 2008) and as a final "clean-up" step to ensure that, among the points visited by some other algorithm, the solution returned is the true efficient set with high probability (for SOSO, see Boesel et al. 2003).

As in Hunter and Nelson (2017) and Pasupathy and Ghosh (2013) for single-objective R\&S, we divide MORS methods into two categories: (1) those with the primary objective of guaranteeing sampling efficiency under some constraint on the total simulation budget $t$, which we call fixedbudget procedures, and (2) those with the primary objective of providing a probabilistic guarantee on the optimality gap of the returned solution, which we call fixed-precision procedures. Fixedbudget MORS procedures are among the most well developed and widely used in all of the existing MOSO literature; a significant proportion of our MORS review consists of fixed-budget procedures.

Since the feasible set may be categorical, in this section only, we work entirely in the objective space and adopt special notation. We consider a finite set of system indices $\mathcal{X}=\{1, \ldots, r\}$, and we refer to the systems in the efficient set as Pareto systems. Since our goal is identification of the Pareto systems and not estimation of their objective vector values, henceforth in this section, the set $\mathcal{P}=\{1, \ldots, p\}$ refers to the set of indices of the true globally Pareto-optimal systems; we drop the use of the term "efficient" and the notation $\mathcal{E}$. The true performance of system $i$ is $\mathrm{g}(i)$, and the true performance of system $i$ on objective $k$ is $g_{k}(i)$. The estimated performance of system $i$ on objective $k$ is $\bar{G}_{k}(i, n):=(1 / n) \sum_{\ell=1}^{n} G_{k \ell}(i)$, where the $\ell$ th simulation replication produces the random vector $G_{\ell}(i):=\left(G_{1 \ell}(i), \ldots, G_{d \ell}(i)\right)$. The estimated objective vector is $\bar{G}(i, n)=$ $\left(\bar{G}_{1}(i, n), \ldots, \bar{G}_{d}(i, n)\right)$. We usually reserve the letter $j$ as an index for the non-Pareto systems.

Now, after expending a finite total simulation budget $t=\sum_{i=1}^{r} n_{i}, n_{i} \geq 1$ for all systems $i \in \mathcal{X}$, a MORS procedure returns the indices of the estimated Pareto systems $\hat{\mathcal{P}}(t):=\{i \in \mathcal{X}: \nexists \ell \in$ $\left.\mathcal{X} \bar{G}\left(\ell, n_{\ell}\right) \leq \bar{G}\left(i, n_{i}\right)\right\}$. If $\hat{\mathcal{P}}(t) \neq \mathcal{P}$, we say that a misclassification (MC) event occurred; this event plays a prominent role in the sections that follow. Adopting the terminology of Hunter and 
McClosky (2016), there are two types of MC events: misclassification by inclusion (MCI) and misclassification by exclusion (MCE). The MCI event occurs when there exists a non-Pareto system $j$ falsely estimated as Pareto, $j \in \hat{\mathcal{P}}(t)$. The MCE event occurs when there exists a Pareto system $i$ falsely estimated as non-Pareto, $i \in \hat{\mathcal{P}}^{c}(t)$. Feldman (2017) writes the MC event as the union of the $\mathrm{MCI}$ event and the MCE event between Pareto systems, called the MCE $\boldsymbol{\rho}$ event:

$$
\mathrm{MC}=[\underbrace{\cup_{j \in \mathcal{P} c} \cap_{\ell \in \mathcal{P}} \cup_{k=1}^{d} \bar{G}_{k}\left(j, n_{j}\right) \leq \bar{G}_{k}\left(\ell, n_{\ell}\right)}_{\text {MCI: a non-Pareto system falsely included }}] \cup[\underbrace{\cup_{i \in \mathcal{P}} \cup_{\ell \in \mathcal{P}} \cap_{k=1}^{d} \bar{G}_{k}\left(\ell, n_{\ell}\right) \leq \bar{G}_{k}\left(i, n_{i}\right)}_{\text {MCE } \mathcal{P}: \text { a Pareto system falsely excludes another }}] .
$$

\subsection{Fixed-Budget MORS Procedures}

Arguably, fixed-budget MORS procedures are the most well-developed, popular, and widely used MOSO methods available. We begin by discussing the first and most popular fixed-budget MORS procedure, MOCBA, followed by two more recent procedures, SCORE and M-MOBA. While most existing procedures fall under the umbrella of MOCBA, SCORE, or M-MOBA, we also refer the reader to Choi and Kim (2018) for a recent fixed-budget procedure based on hypothesis testing.

Since fixed-budget MORS procedures frequently derive a simulation budget allocation rule by first assuming all required parameters are known, we present the rules for each method in terms of the true unknown parameters. Since the true unknown parameters can only be estimated, the allocation rules we present can be implemented in a sequential sampling framework that broadly consists of the following steps: (1) obtain $n_{0} \geq 2$ simulation replications from each system; (2) estimate the parameters of the sampling rule and select the system(s) from which to take the next $\Delta \geq 1$ simulation replications according to the estimated version of the sampling rule; and (3) obtain the required simulation replications from the selected systems and, unless the termination criterion is met, go to Step (2). Each round of obtaining the next $\Delta$ simulation replications is frequently called a "stage." For convergence, the sampling rule should ensure that each system is sampled infinitely often. The termination criterion is usually that the predetermined total simulation budget $t$ has been expended, at which time the set of estimated Pareto systems $\hat{\mathcal{P}}(t)$ is returned to the user.

The fixed-budget MORS procedures we discuss derive simulation budget allocation rules under one or more assumptions: (1) the normality assumption, which is that the simulation replications from system $i, G_{1}(i), \ldots, G_{n_{i}}(i)$, are independent and identically distributed (i.i.d.) normal random vectors with mean $\mathbf{g}(i)$ and covariance matrix $\Sigma(i)$, where $\sigma_{k}^{2}(i)$ is the $k$ th diagonal entry, or (2) the independent objectives assumption, which is that $\Sigma(i)$ is diagonal. While these assumptions may seem restrictive, in general, such rules constitute a model to guide the simulation budget allocation; procedures may be robust to violations of these assumptions (see, e.g., Pasupathy et al. 2015).

3.1.1 MOCBA. The Multiobjective Optimal Computing Budget Allocation (MOCBA) framework (Lee et al. 2010) is a multiobjective version of the popular Optimal Computing Budget Allocation (OCBA) framework (Chen et al. 2000) for $d \geq 2$ objectives. The goal of MOCBA is to provide a simulation budget allocation rule that minimizes the probability of MC. Since this probability is difficult to minimize directly, under the normality and independent objectives assumptions, Lee et al. (2010) derive upper bounds $a e_{1}, a e_{2}$ such that $\mathbb{P}\{\mathrm{MCI}\} \leq a e_{1}$ and $\mathbb{P}\{\mathrm{MCE}\} \leq a e_{2}$. Then, they formulate an optimization problem that minimizes the largest bound:

$$
\text { Find: } \operatorname{argmin}_{\left(n_{1}, n_{2}, \ldots, n_{r}\right)} \max \left\{a e_{1}, a e_{2}\right\} \text { s.t. } n_{1}+n_{2}+\cdots+n_{r} \leq t .
$$

Depending on which bound is larger, two allocation rules result: the $a e_{1}$ rule and the $a e_{2}$ rule.

Before we present the $a e_{1}$ and $a e_{2}$ rules, we require additional notation. In the MOCBA framework, systems play one of two roles: dominating or dominated. In the $a e_{1}$ and $a e_{2}$ rules, the 


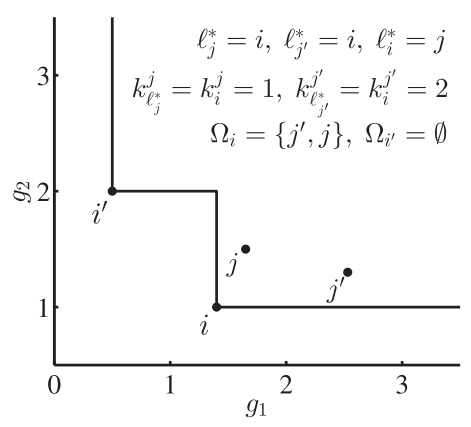

Fig. 3. MOCBA: The figure shows systems $i, i^{\prime}$ in a dominating role and $j, j^{\prime}$ in a nondominating role under independent objectives and equal variances.

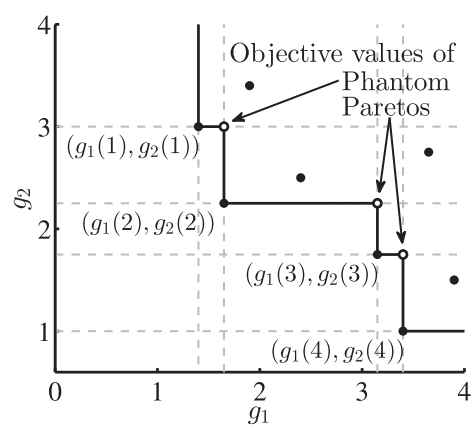

Fig. 4. SCORE: The figure shows Pareto systems 1, 2, 3, and 4 and the objective values of three phantom Pareto systems.

allocation to each system is determined by its role. Loosely speaking, a system $j$ in a dominated role receives an allocation $n_{j}=t \alpha_{j}$ for $\alpha_{j} \in[0,1]$ that is inversely proportional to its scaled squared "distance" from the system most likely to dominate it, where "distance" is the difference in objective values on the objective least likely to allow that system to dominate system $j$. To write the allocations precisely, for any system $j$, let $\ell_{j}^{*}$ be the system most likely to dominate system $j$. For any two systems $i$ and $j$, let $k_{i}^{j}$ be the objective on which system $i$ is least likely to dominate system $j$. Then intuitively, if system $\ell_{j}^{*}$ is the system most likely to dominate $j$, then objective $k_{\ell_{j}^{*}}^{j}$ is the objective that is most likely to prohibit system $\ell_{j}^{*}$ from dominating system $j$. An example system configuration is shown in Figure 3. For brevity, define $\delta(j, \ell, k):=g_{k}(j)-g_{k}(\ell)$. Then the allocation to a system $j^{\prime}$ in a dominated role, relative to another system $j$ in a dominated role, is

$$
\frac{\alpha_{j^{\prime}}}{\alpha_{j}}=\left(\delta^{2}\left(j, \ell_{j}^{*}, k_{\ell_{j}^{*}}^{j}\right) /\left[\sigma_{k_{\ell_{j}^{*}}^{j}}^{2}(j)+\sigma_{k_{\ell_{j}^{*}}^{j}}^{2}\left(\ell_{j}^{*}\right) / \gamma_{j}\right]\right) /\left(\delta^{2}\left(j^{\prime}, \ell_{j^{\prime}}^{*}, k_{\ell_{j^{\prime}}^{j^{\prime}}}^{j^{\prime}}\right) /\left[\sigma_{k_{\ell_{j^{\prime}}^{j^{\prime}}}^{2}}^{2}\left(j^{\prime}\right)+\sigma_{k_{\ell_{j^{\prime}}^{j^{\prime}}}^{2}}^{2}\left(\ell_{j^{\prime}}^{*}\right) / \gamma_{j^{\prime}}\right]\right),
$$

where $\gamma_{j}=\alpha_{\ell_{j}^{*}} / \alpha_{j}$ and $\gamma_{j^{\prime}}=\alpha_{\ell_{j^{\prime}}^{*}} / \alpha_{j^{\prime}}$. Assuming that $\ell_{j}^{*}$ and $\ell_{j^{\prime}}^{*}$ are Pareto systems and $j$ and $j^{\prime}$ are non-Pareto systems with $\alpha_{\ell_{j}^{*}} \gg \alpha_{j}$ and $\alpha_{\ell_{j^{\prime}}^{*}} \gg \alpha_{j^{\prime}}$, then $\gamma_{j}$ and $\gamma_{j^{\prime}}$ are large, and our previously stated intuition regarding these relative allocations holds approximately. To write the allocation to systems $i$ in a dominating role, we require additional notation. First, denote the allocation to any system $\ell$ as $\alpha_{\ell}=\beta_{\ell} / \sum_{\ell^{\prime}=1}^{r} \beta_{\ell^{\prime}}$, where for all systems $j$ in a dominated role, the value of $\beta_{j}$ can be obtained from Equation (1). For systems $i$ in a dominating role, define $\beta_{i}:=\sqrt{\sum_{j \in \Omega_{i}}\left[\sigma_{k_{i}^{j}}^{2}(i) / \sigma_{k_{i}^{j}}^{2}(j)\right] \beta_{j}^{2}}$, where $\Omega_{i}$ is the set of all systems $j$ playing a dominated role in which system $i$ is the system most likely to dominate system $j$. If $\Omega_{i}$ is empty, then the allocation to system $i$ is $\alpha_{i}=0$.

We now present the $a e_{1}$ and $a e_{2}$ rules. First, if $a e_{1}>a e_{2}$, Lee et al. (2010) are most concerned that a non-Pareto system will be falsely included in the estimated Pareto set. Therefore, in the $a e_{1}$ rule, non-Pareto systems play the dominated role, and Pareto systems play the dominating role. In the $a e_{2}$ rule, $a e_{1} \leq a e_{2}$, so Lee et al. (2010) are most concerned that a Pareto system will be falsely excluded from the estimated Pareto set. Thus, all non-Pareto systems play the dominating role, while some Pareto systems play the dominating role and others play the dominated role. Loosely speaking, the set of Pareto systems that play the dominated role, $\mathcal{S}_{A} \subseteq \mathcal{P}$, is the set of all Pareto systems that are more likely to be dominated than to dominate another Pareto; $\mathcal{S}_{A}$ can be empty. 
Lee et al. (2010) show that as the total simulation budget $t$ tends to infinity, the $a e_{1}$ and $a e_{2}$ rules asymptotically minimize the $a e_{1}$ and $a e_{2}$ bounds, respectively. The MOCBA framework consists of sequentially estimating the $a e_{1}$ and $a e_{2}$ bounds, selecting a sampling rule based on the largest estimated bound, and implementing an estimated version of the selected rule to obtain the next $\Delta$ simulation replications. Teng et al. (2010) modify MOCBA to allow an indifference zone, which is the smallest difference in objective values the decision maker wishes to detect. Also, Li et al. (2018) present three new versions of MOCBA.

3.1.2 SCORE. The Sampling Criteria for Optimization using Rate Estimators (SCORE) framework, introduced by Pasupathy et al. (2015), is a simulation budget allocation framework that allocates to suboptimal systems in inverse proportion to their squared standardized optimality gaps. The SCORE allocations result from a limiting regime in which the number of suboptimal or non-Pareto systems are sent to infinity. The Pareto systems, which receive many more samples than the non-Pareto systems in this limiting regime, receive a heuristically determined allocation.

Under the normality assumption, Feldman and Hunter (2018) derive SCORE allocations for biobjective R\&S that account for correlation between the objectives. To write the biobjective SCORE allocations, we first require the concept of phantom Pareto systems, introduced by Hunter and McClosky (2016). Phantom Pareto systems are constructed from, and exist in relation to, the objective values of the Pareto systems. They make the mathematical analysis of the $\mathbb{P}\{M C\}$ tractable by writing the MCI event, which contains dependence, in an MCE-like fashion. That is, assuming the Pareto set is fixed and known, a non-Pareto system is falsely included in the Pareto set if and only if it dominates a phantom Pareto system. To define these systems in the biobjective case, first label the Pareto systems from smallest to largest on objective 1 , so that $g_{1}(1)<g_{1}(2)<\cdots<g_{1}(p)$. Because there are two objectives, this ordering implies $g_{2}(1)>g_{2}(2)>\cdots>g_{2}(p)$. Then the $p+1$ phantom Pareto systems are defined as having objective values $\left(g_{1}(i+1), g_{2}(i)\right)$ for all Pareto systems $i=1, \ldots, p-1$ if $p \geq 2$, in addition to two phantom Pareto systems placed at $\left(g_{1}(1), \infty\right)$ and $\left(\infty, g_{2}(p)\right)$. An example set of phantom Pareto systems is shown in Figure 4. Letting $g_{1}(p+1):=$ $\infty, g_{2}(0):=\infty$, and letting $\ell$ index the phantom Pareto systems, define the score of a non-Pareto system $j$ as

$$
\mathbb{S}_{j}:=\min _{\ell \in\{0, \ldots, p\}}\left\{\inf _{x \leq g_{1}(\ell+1), y \leq g_{2}(\ell)} \frac{1}{2\left(1-\rho_{j}^{2}\right)}\left(\frac{\left(x-g_{1}(j)\right)^{2}}{\sigma_{1}^{2}(j)}-\frac{2 \rho_{j}\left(x-g_{1}(j)\right)\left(y-g_{2}(j)\right)}{\sigma_{1}^{2}(j) \sigma_{2}^{2}(j)}+\frac{\left(y-g_{2}(j)\right)^{2}}{\sigma_{2}^{2}(j)}\right)\right\},
$$

where $\rho_{j} \in(-1,1)$ is the correlation between the objectives for system $j$. Thus, the score is the minimum squared standardized "distance" from the non-Pareto system to the phantom Pareto systems in the objective function space. Then the relative allocations for non-Pareto systems are $\alpha_{j^{\prime}} / \alpha_{j}=\mathbb{S}_{j} / \mathbb{S}_{j^{\prime}}$ for all $j, j^{\prime} \in \mathcal{P}^{c}, j \neq j^{\prime}$. These allocations can be compared with Equation (1).

Feldman and Hunter (2018) suggest solving for the allocation to the Pareto systems in a way that controls the probabilities of both MCI and MCE events and requires solving a convex optimization problem whose complexity depends only on the number of Pareto systems. They show that solving for the biobjective SCORE allocations is fast for up to 10,000 systems. These allocations are incorporated into a sequential framework for implementation.

As in the biobjective case, multiobjective SCORE allocations for $d \geq 3$ can be specified by defining phantom Pareto systems in higher dimensions. Applegate et al. (2018) address this topic in a working paper; preliminary work is published in Feldman (2017) and Feldman et al. (2015).

3.1.3 M-MOBA. We now discuss two multiobjective extensions of the small-sample Expected Value of Information procedures of Chick et al. (2010) called Myopic Multi-Objective Budget Allocation (M-MOBA) (Branke and Zhang 2015) and M-MOBA-HV (Branke et al. 2016). Under the normality and independent objectives assumptions, and in the context of a sequential sampling 
framework, both M-MOBA and M-MOBA-HV adopt a Bayesian perspective to calculate the "best" system from which to obtain an additional $\Delta \geq 1$ samples in the next stage. In the case of M-MOBA, the best system is the one that has the highest probability of changing the observed Pareto set. In the case of M-MOBA-HV, the best system is the one that leads to the largest change in the observed expected hypervolume (see definitions in Section 2.3.2). That is, for each system $i$, M-MOBA-HV requires calculating the expected hypervolume difference between the current observed Pareto set and the Pareto set that would result from obtaining the next $\Delta$ samples from system $i$. All $\Delta$ samples in the next stage are allocated to the system, resulting in the largest expected hypervolume difference.

Branke et al. (2016) argue that M-MOBA-HV is more likely to be relevant for decision makers, for the following reasons. Since the goal of MOCBA, SCORE, and M-MOBA is to efficiently allocate samples in such a way that the probability of an MC event is low, these frameworks are likely to expend a significant amount of the simulation budget attempting to distinguish between systems "near" the Pareto set. The goal of M-MOBA-HV, however, is to allocate the simulation budget in such a way that the estimated expected hypervolume is "close" to the true expected hypervolume. Therefore, M-MOBA-HV may expend more effort estimating the objective values of clearly Pareto systems than distinguishing the minor differences between Pareto and nearly Pareto systems.

\subsection{Fixed-Precision MORS Procedures}

Fixed-precision MORS procedures are notably underdeveloped in the literature, despite the popularity of analogous procedures for single-objective R\&S. To the best of our knowledge, the only work in this area so far is by Batur and Choobineh (2010), Lee (2014, Ch. 4), and Wang and Wan (2017). Batur and Choobineh (2010) present a fixed-precision procedure designed for biobjective R\&S where the objectives are the mean and variance of the system performances. Lee (2014, Ch. 4) provides three general fixed-precision MORS procedures based on an indifference-zone formulation, where the indifference zone on objective $k$ is the smallest difference in system performances that a decision maker cares to detect. Wang and Wan (2017) present preliminary work on a general, fully sequential fixed-precision MORS procedure that is based on the generalized sequential probability ratio test. In Wang and Wan (2017), when the procedure terminates after expending a random total simulation budget $T$, the desired guarantee is that the probability of an MC event is small; that is, $\mathbb{P}\{\hat{\mathcal{P}}(T) \neq \mathcal{P}\} \leq \alpha$ for a user-defined error probability bound $\alpha$. We remark that Chen and Lee (2009) present a MORS procedure based on the Enhanced Two-Stage Selection (ETSS) procedures of Chen and Kelton (2005), and thus provide an approximate probabilistic guarantee on the quality of the returned solution. In Section 7, we speculate on the future development of fixed-precision MORS procedures.

\section{CONTINUOUS MULTIOBJECTIVE SIMULATION OPTIMIZATION}

In this section, we consider MOSO problems with continuous decision variables. Since the efficient and Pareto sets may be uncountable, methods to solve Problem $M$ implemented with a finite total simulation budget $t$ may directly identify only a finite number of estimated efficient points that, ideally, provide an accurate representation of the Pareto set in the objective function space. As the total simulation budget $t$ tends to infinity, we would like MOSO methods to provide some form of probabilistic guarantee on the convergence of the returned solution to a local efficient set. The work on theory, methods, and algorithms for continuous MOSO with guaranteed convergence consists primarily of methods that employ a sample average approximation (SAA) framework. We discuss multiobjective SAA in Section 4.1, SAA under scalarization in Section 4.2, and continuous MOSO algorithms that use an SAA framework in Section 4.3. We refer the reader to Pang et al. 
(2018) for a recent paper on SAA with multiple objectives and parametric variational inequality constraints. Also, we remark that Villarreal-Marroquín et al. $(2011,2013)$ provide metamodel-based approaches for continuous MOSO but do not discuss convergence topics. Thus, we do not provide details about these methods.

\subsection{Sample Average Approximation for Continuous MOSO}

Popular in the SOSO context, SAA provides a way of estimating the efficient and Pareto sets by solving the deterministic sample-path problem for a fixed simulation budget $n$. SAA replaces Problem $M$ with the corresponding sample-path problem:

Problem $\bar{M}: \underset{\mathbf{x} \in \mathcal{X}}{\operatorname{minimize}}\left\{\bar{G}(\mathbf{x}, n)=\left(\bar{G}_{1}(\mathbf{x}, n), \ldots, \bar{G}_{d}(\mathbf{x}, n)\right):=\left(\frac{1}{n} \sum_{i=1}^{n} G_{1}\left(\mathbf{x}, \xi_{i}\right), \ldots, \frac{1}{n} \sum_{i=1}^{n} G_{d}\left(\mathbf{x}, \xi_{i}\right)\right)\right\}$.

Notice that sample-path versions of all optimal points and sets from Section 2.1 can be defined by replacing the true function values with the estimators $\bar{G}(\mathbf{x}, n)$. For brevity, we define only the global solution to the sample-path Problem $\bar{M}$, the sample-path efficient set $\overline{\mathcal{E}}(n):=\left\{X^{*} \in \mathcal{X}: \nexists X \in \mathcal{X} \ni\right.$ $\left.\bar{G}(X, n) \leq \bar{G}\left(X^{*}, n\right)\right\}$, and its image, the sample-path Pareto set $\overline{\mathcal{P}}(n):=\left\{\bar{G}\left(X^{*}, n\right): X^{*} \in \overline{\mathcal{E}}\right\}$. The analogous definition of the sample-path weakly efficient set $\overline{\mathcal{E}}^{w}(n)$ is implied.

SAA is an algorithmic framework and not an algorithm, since a method for solving the samplepath Problem $\bar{M}$ is not defined. Consequently, there is more than one potential interpretation of the simulation budget $n$ as it relates to the total simulation budget $t$. If the entire vector function $\bar{G}(\cdot, n)$ is observed for one realization of $\xi_{i}, i=1, \ldots, n$, then it may follow that $n=t$. For example, in the single-objective context of the continuous news vendor problem, the entire estimated profit function is observed for a single realization of demands $\xi_{i}, i=1, \ldots, n$ (see, e.g., Kim et al. 2015, p. 208). Alternatively, an algorithm operating in an SAA framework may query an oracle for $n$ simulation replications at each visited point in the feasible set, so that $t \geq n$. In this case, using the same sample size at every point facilitates the use of common random numbers (CRNs) (see Law 2015, p. 588). Since the simulation budget expended at each $\mathbf{x}$ is nevertheless $n$, in the context of SAA, we explicitly denote the dependence of sample-path optimal points and sets on $n$ instead of $t$.

Under appropriate regularity conditions, as the simulation budget $n$ increases to infinity, ideally, some metric of difference between the sample-path solution to Problem $\bar{M}$ and the true solution to Problem $M$ converges to zero w.p.1. Bonnel and Collonge (2014) provide regularity conditions and convergence results regarding the Hausdorff distance between the sample-path Pareto set and the true Pareto set, as well as the Hausdorff distance between the sample-path weakly efficient set and the true weakly efficient set. To present the key results of Bonnel and Collonge (2014), we first describe the required assumptions; a subset of these assumptions are needed for each result.

Assumption 1 (Bonnel And Collonge 2014, P. 411). We assume one or more of the following:

(1) $\mathcal{X}$ is a nonempty and compact subset of $\mathbb{R}^{q}$.

(2) The random variables $\xi_{i}, i=1,2, \ldots$ are i.i.d. with $P\left\{\xi_{i} \in \Xi\right\}=1$ for all $i=1,2, \ldots$.

(3) For each objective $k=1, \ldots, d$, the function $G_{k}(\mathbf{x}, \xi)$ is finite valued and continuous on $\mathcal{X}$ for a.e. $\xi \in \Xi$.

(4) For each objective $k=1, \ldots, d$, the function $G_{k}(\mathbf{x}, \xi)$ is dominated by an integrable function $\kappa_{k}$, that is, $\mathbb{E}_{\xi}\left[\kappa_{k}(\cdot)\right]<\infty$ and $\left|G_{k}(\mathbf{x}, \xi)\right| \leq \kappa_{k}(\xi)$ for all $\mathbf{x} \in \mathcal{X}$ and a.e. $\xi \in \Xi$.

(5) $X$ is convex.

(6) For each objective $k=1, \ldots, d, G_{k}(\mathbf{x}, \xi)$ is strictly convex on $\mathcal{X}$ for a.e. $\xi \in \Xi$. 
In Assumptions 1(3), 1(4), and 1(6), "for a.e. $\xi \in \Xi$ " means that the assertion holds for almost every $\xi \in \Xi$, that is, w.p.1.

Under Assumptions 1(1) through 1(4), for each objective $k \in\{1, \ldots, d\}, g_{k}(\mathbf{x})$ is finite valued and continuous on $\mathcal{X}$, and the uniform LLN holds (Shapiro 2003, p. 363, Proposition 7). Recall that the uniform LLN states that a sequence of functions $\bar{G}(\mathbf{x}, n)$ converges to $g(\mathbf{x})$ w.p.1. as $n \rightarrow \infty$, uniformly on $\mathbf{x} \in \mathcal{X}$ if and only if $\mathbb{P}\left\{\lim _{n \rightarrow \infty} \sup _{\mathbf{x} \in \mathcal{X}}|\bar{G}(\mathbf{x}, n)-g(\mathbf{x})|=0\right\}=1$. By the uniform LLN, the following result regarding the maximum Euclidean distance between $\bar{G}(\mathbf{x}, n)$ and the objective vector $\mathbf{g}(\mathbf{x})$ holds (see Bonnel and Collonge 2014, p. 412; Fliege and Xu 2011, p. 151).

Proposition 4.1. Under Assumptions 1(1) through 1(4), $\mathbb{P}\left\{\lim _{n \rightarrow \infty} \sup _{\mathbf{x} \in \mathcal{X}}\|\bar{G}(\mathbf{x}, n)-\mathbf{g}(\mathbf{x})\|=\right.$ $0\}=1$.

Under equally mild conditions, Bonnel and Collonge (2014) show that, as $n$ goes to infinity, the Hausdorff distance between the sample-path Pareto set $\overline{\mathcal{P}}(n)$ and the true Pareto set $\mathcal{P}$ goes to zero w.p.1, and the distance between the sample-path weakly efficient set $\overline{\mathcal{E}}^{w}(n)$ and the true weakly efficient set $\mathcal{E}^{w}$ goes to zero w.p.1.

Theorem 4.2 (Bonnel And Collonge 2014, P. 417, 421). Under Assumptions 1(1) through 1(4),

(1) the Hausdorff distance between the sample-path Pareto set $\overline{\mathcal{P}}(n)$ and the true Pareto set $\mathcal{P}$ goes to zero as $n \rightarrow \infty$ w.p. 1 , that is, $\mathbb{P}\left\{\lim _{n \rightarrow \infty} \mathbb{H}(\overline{\mathcal{P}}(n), \mathcal{P})=0\right\}=1$; and

(2) the distance between the sample-path weakly efficient set $\overline{\mathcal{E}}^{w}(n)$ and the true weakly efficient set $\mathcal{E}^{w}$ goes to zero as $n \rightarrow \infty$ w.p. 1 , that is, $\mathbb{P}\left\{\lim _{n \rightarrow \infty} \mathbb{D}\left(\overline{\mathcal{E}}^{w}(n), \mathcal{E}^{w}\right)=0\right\}=1$.

To ensure no weakly efficient points are "missed," the feasible region $\mathcal{X}$ must be convex, and each $G_{k}(\mathbf{x}, \xi)$ must be strictly convex on $\mathcal{X}$ for a.e. $\xi \in \Xi$. Recall from Section 2.1.3 that if Problem $M$ is convex, then the local efficient set is equal to the global efficient set.

Theorem 4.3 (Bonnel And Collonge 2014, P. 417). Under Assumptions 1(1) through 1(6), the Hausdorff distance between the sample-path weakly efficient set $\overline{\mathcal{E}}^{w}(n)$ and the true weakly efficient set $\mathcal{E}^{w}$ goes to zero as $n \rightarrow \infty$ w.p.1, that is, $\mathbb{P}\left\{\lim _{n \rightarrow \infty} \mathbb{H}\left(\overline{\mathcal{E}}^{w}(n), \mathcal{E}^{w}\right)=0\right\}=1$.

Thus, Bonnel and Collonge (2014) provide general consistency results for SAA in the multiobjective context. We remark here that Kim and Ryu (2011a) discuss the uniform convergence of gradient estimates in the context of SAA for MOSO. Despite the more specific focus of their papers overall, Bonnel and Collonge (2015) and Fliege and Xu (2011) also provide some general convergence rate results for SAA.

\subsection{SAA for Continuous MOSO Under Scalarization}

Algorithms for approximating the efficient set may employ scalarization techniques in an SAA framework, such as the trust-region algorithm of Kim and Ryu (2011a) discussed in the next section. To show convergence of such methods, consistency results are required for SAA under scalarization. We now discuss three such scalarization approaches: the linear weighted sum, the smoothing Chebyshev scalarization, and the single-objective product formulation.

4.2.1 Linear Weighted Sum. The linear weighted sum method minimizes the sum of the weighted objectives. Given weights $\mathbf{w}=\left(w_{1}, \ldots, w_{d}\right) \in \mathbb{R}^{d}, \mathbf{w} \geq \mathbf{0}_{d}, \sum_{k=1}^{d} w_{k}=1$, define

$$
\text { Problem } S_{\text {linear }}(\mathbf{w}): \text { minimize }_{\mathbf{x} \in \mathcal{X}} \sum_{k=1}^{d} w_{k} g_{k}(\mathbf{x}) \text {. }
$$

If the weights are positive, $\mathbf{w}>\mathbf{0}_{d}$, then the solution to Problem $S_{\text {linear }}(\mathbf{w})$ is efficient (Miettinen 1999, p. 78, Theorem 3.1.2). If Problem $M$ is convex, then for each efficient point $\mathbf{x}^{*} \in \mathcal{E}$, there exists a weight vector $\mathbf{w}$ such that $\mathbf{x}^{*}$ is a solution to Problem $S_{\text {linear }}(\mathbf{w})$ (Miettinen 1999, p. 79, 
Theorem 3.1.4). If Problem $M$ is nonconvex, there may be points in the efficient set that no weight vector can retrieve; these points are the preimage of points in the Pareto set for which no supporting hyperplane exists (see Section 2.1.3). Marler and Arora (2004, p. 389) consider the linear weighted sum method a benchmark approach, since it is among the most common and easy-toimplement scalarization approaches.

The SAA version of Problem $S_{\text {linear }}(\mathbf{w})$ is

$$
\text { Problem } \bar{S}_{\text {linear }}(\mathbf{w}, n): \text { minimize } \mathbf{x}_{\mathbf{x}} \mathcal{X} \sum_{k=1}^{d} w_{k} \bar{G}_{k}(\mathbf{x}, n) \text {. }
$$

Bonnel and Collonge (2014) use the linear weighted sum method to prove consistency of the sample-path weakly efficient set presented in Theorem 4.3 and, in doing so, implicitly provide consistency results for SAA using the linear weighted sum method under Assumptions 1(1) through $1(6)$.

4.2.2 Smoothing Chebyshev Scalarization. Fliege and Xu (2011) propose and analyze the properties of a smoothing Chebyshev scalarization in the context of SAA. Given weights w defined in the previous section and a fixed dominating reference vector $\mathrm{g}^{\text {ref }}$, such as $\mathrm{g}^{\text {ideal }}$, the weighted Chebyshev $\left(L_{\infty}\right.$-norm) scalarization is

$$
\text { Problem } S_{\infty}(\mathbf{w}): \operatorname{minimize}_{\mathbf{x} \in \mathcal{X}} \max _{k \in\{1, \ldots, d\}} w_{k}\left|g_{k}(\mathbf{x})-g_{k}^{\text {ref }}\right| \text {. }
$$

Unlike the linear weighted sum, the weighted Chebyshev scalarization can retrieve all Pareto points by varying the parameter w, even for nonconvex problems (Miettinen 1999, pp. 97-99). However, if we wish to solve Problem $M$ using weighted Chebyshev scalarization, the resulting Problem $S_{\infty}(\mathbf{w})$ is nonsmooth due to the maximum operation. Several reformulations of Chebyshev scalarization exist to make the optimization problem smooth. Fliege and $\mathrm{Xu}$ (2011) propose a smoothing Chebyshev scalarization, defined as

$$
\text { Problem } S_{\infty}^{\text {smooth }}(\mathbf{w}, s): \text { minimize }_{\mathbf{x} \in X} p\left(\left(w_{1}\left(g_{1}(\mathbf{x})-g_{1}^{\mathrm{ref}}\right), \ldots, w_{d}\left(g_{d}(\mathbf{x})-g_{d}^{\mathrm{ref}}\right)\right), s\right),
$$

where the function $p(\mathrm{y}, s):=s \log \sum_{k=1}^{d} e^{y_{k} / s}$ for fixed $s>0$ is continuously differentiable and strictly convex in $\mathbf{y}$, and $p(\mathbf{y}, 0):=0$. Fliege and Xu (2011) assert that solving Problem $S_{\infty}^{\text {smooth }}(\mathbf{w}, s)$ for fixed $(\mathbf{w}, s)$ yields an efficient point, and the set of solutions to Problem $S_{\infty}^{\text {smooth }}(\mathbf{w}, s)$ obtained across all $\mathbf{w}$ approximates the set of solutions to Problem $S_{\infty}(\mathbf{w})$ obtained across all $\mathbf{w}$ as $s \rightarrow 0$.

To study the smoothed Chebyshev scalarization in an SAA framework, Fliege and Xu (2011) formulate the SAA version of Problem $S_{\infty}^{\text {smooth }}(\mathbf{w}, s)$ as

$$
\text { Problem } \bar{S}_{\infty}^{\text {smooth }}(\mathbf{w}, s, n): \text { minimize }_{\mathbf{x} \in \mathcal{X}} p\left(\left(w_{1}\left(\bar{G}_{1}(\mathbf{x}, n)-g_{1}^{\mathrm{ref}}\right), \ldots, w_{d}\left(\bar{G}_{d}(\mathbf{x}, n)-g_{d}^{\mathrm{ref}}\right)\right), s\right) .
$$

Under appropriate regularity conditions, Fliege and $\mathrm{Xu}$ (2011) prove consistency results and convergence rate results as a function of the parameter $s$.

4.2.3 Single-Objective Product Formulation. Kim and Ryu (2011a) discuss the convergence properties of the sample-path efficient and Pareto sets under the single-objective product formulation. The single-objective product formulation is a scalarization introduced by Audet et al. (2008) that results in an optimization problem with desirable properties. For example, under mild regularity conditions, the scalarized objective function is locally Lipschitz continuous and differentiable whenever those properties are held by the functions comprising the vector $\mathbf{g}$. The scalarization is

$$
\text { Problem } S_{\text {prod }}\left(\mathbf{g}^{\mathrm{REF}}\right): \operatorname{minimize}_{\mathbf{x} \in X}\left\{\phi\left(\mathbf{x}, \mathbf{g}^{\mathrm{REF}}\right):=-\prod_{k=1}^{d}\left(\max \left\{g_{k}^{\mathrm{REF}}-g_{k}(\mathbf{x}), 0\right\}\right)^{2}\right\},
$$

where $\mathrm{g}^{\mathrm{REF}}$ is a dominated reference point. Audet et al. (2008) show that if $\mathbf{x}^{*}$ is a solution to Problem $S_{\text {prod }}\left(\mathrm{g}^{\mathrm{REF}}\right)$ with $\phi\left(\mathbf{x}, \mathrm{g}^{\mathrm{REF}}\right)<0$, then $\mathbf{x}^{*}$ is an efficient point. Thus, multiple points in the efficient set can be found by varying the reference point $\mathrm{g}^{\mathrm{REF}}$ and solving each scalarized problem. 
Kim and Ryu (2011a) formulate an SAA version of this scalarization as

Problem $\bar{S}_{\text {prod }}\left(\mathbf{g}^{\mathrm{REF}}, n\right): \operatorname{minimize}_{\mathbf{x} \in \mathcal{X}}\left\{\bar{\phi}\left(\mathbf{x} ; \mathbf{g}^{\mathrm{REF}}\right):=\frac{1}{n} \sum_{i=1}^{n}\left[-\prod_{k=1}^{d}\left(\max \left\{g_{k}^{\mathrm{REF}}-G_{k}\left(\mathbf{x}, \xi_{i}\right), 0\right\}\right)^{2}\right]\right\}$.

Under regularity conditions including Assumptions 1(1) through 1(4), the uniform LLN holds for $\bar{\phi}\left(\cdot, \mathrm{g}^{\mathrm{REF}}\right)$ (Kim and Ryu 2011a). Thus, standard results from the SAA literature apply to $\bar{\phi}\left(\cdot, \mathrm{g}^{\mathrm{REF}}\right)$.

\subsection{SAA-Based Algorithms for Continuous MOSO}

Few algorithms exist for continuous MOSO in the literature. In the following sections, we discuss three algorithms based on an SAA framework. Notice that when we discuss specific SAA-based algorithms, we may not solve each sample-path problem to optimality. Thus, the estimated efficient set, $\hat{\mathcal{E}}(n)$, may not be equal to the true sample-path efficient set $\overline{\mathcal{E}}(n)$.

4.3.1 Smoothed Chebyshev with Equidistant or Random Weights. The theory of Fliege and Xu (2011) focuses on characterizing the entire efficient set for $d \geq 2$ objectives, but the authors do not propose a corresponding algorithm as such. The following algorithm could be deduced from their paper and numerical results: (1) select a finite set of equidistant or randomly chosen weights, a parameter $s>0$, a sample size $n$, and initial starting points for each scalarized problem; (2) solve each Problem $\bar{S}_{\infty}^{\text {smooth }}(\mathbf{w}, s, n)$ using a deterministic single-objective optimization routine; and (3) construct an estimated efficient set $\hat{\mathcal{E}}(n)$ from the set of returned solutions to the problems solved in Step (2). To choose parameters, Fliege and Xu (2011) remark on p. 147 that if Problem $M$ is convex, the parameter $s$ need not be small, and a "sufficiently good discretization" of the weight space results in a good approximation of the Pareto set. This property is not necessarily true for all scalarizations. For example, Das and Dennis (1997) explain how choosing evenly spaced scalarization parameters does not necessarily produce evenly spaced points in the Pareto set in the context of the linear weighted sum method.

4.3.2 Trust-Region Methods. Kim and Ryu (2011a, 2011b) provide a derivative-free trust-region algorithm for $d=2$ stochastic objectives that employs the single-objective product formulation in an SAA framework. This algorithm can be considered a stochastic version of the deterministic derivative-free biobjective trust-region method in Ryu and Kim (2014), which provably converges to a collection of first-order critical points of one of the two objectives or of the scalarized objective. Here, "derivative free" implies that derivative information is not automatically returned by the simulation oracle along with the estimated function values; derivative information must be explicitly constructed from function value estimates. Given a value of $n$, after initialization, the algorithm in Kim and Ryu (2011a) iterates through the following general steps: (1) select the most "isolated" point $X$ in a set of estimated efficient points from the previous iteration; (2) create surrogate quadratic models for each objective and the scalarized single-objective product formulation in a trust region around $X$, where the trust region is the region in which we trust the surrogate model; (3) solve three single-objective subproblems in the trust region, where the objective in each subproblem is specified by a surrogate model; and (4) update algorithm parameters including the trust-region radius, update the estimated efficient set $\hat{\mathcal{E}}(n)$, and go to the next iteration. The algorithm by Kim and Ryu (2011a) is designed to find a good representation of a local efficient set.

4.3.3 MOROZS. Wang (2017) develops the derivative-based multiobjective retrospective optimization using the zigzag search (MOROZS) algorithm for $d=2$ stochastic objectives, assuming all local efficient points are global efficient points. Notice that here, the term "derivative based" implies that derivative estimates are automatically supplied by the oracle along with each function value estimate. MOROZS is a stochastic version of the deterministic derivative-based zigzag 
search (ZS) algorithm of Wang (2013) that is implemented in a retrospective approximation (RA) framework.

RA is a version of SAA in which a sequence of sample-path problems are solved, each at an increasing sample size. Thus, Problem $\bar{M}$ is solved repeatedly for the increasing sample size sequence $\left\{n_{v}, v=1,2, \ldots\right\}$, and the solution to the $(v-1)$ th sample-path problem is used as a warm start to the $v$ th sample-path problem. Loosely speaking, RA ensures sampling efficiency by using small sample sizes when the algorithm is "far away" from a truly optimal point and by using larger sample sizes only when the algorithm is "closer" to a truly optimal point; see Pasupathy and Ghosh (2013, pp. 143-145) for a more detailed discussion.

Then, instead of implementing ZS on Problem $\bar{M}$ directly for a single sample size $n$, MOROZS uses ZS to repeatedly solve Problem $\bar{M}$ for a sequence of increasing sample sizes $\left\{n_{v}, v=1,2, \ldots\right\}$. The estimated efficient points from the $(v-1)$ th iteration are used as warm starts for ZS in the $v$ th iteration of MOROZS. Because of its reliance on ZS, MOROZS is designed to find efficient points that are "evenly spaced" in the Pareto set.

\section{INTEGER-ORDERED MULTIOBJECTIVE SIMULATION OPTIMIZATION}

In this section, we consider solving Problem $M$ when the decision variable space is integer-ordered, and hence $X \subseteq \mathbb{Z}^{q}$. In this context, both the efficient set and the Pareto set are countable; if they are also finite, an algorithm may attempt to identify an entire local efficient set. As in Wang et al. (2013), we define a flexible neighborhood structure based on Euclidean distance for use in the definitions of local optimality in Section 2.1.2.

Definition 5.1. For $a \geq 0$, the $\mathcal{N}_{a}$-neighborhood of the feasible decision point $\mathbf{x} \in X \subseteq \mathbb{Z}^{q}$ is $\mathcal{N}_{a}(\mathbf{x}):=\left\{\mathbf{x}^{\prime} \in \mathbb{Z}^{q}:\left\|\mathbf{x}-\mathbf{x}^{\prime}\right\| \leq a\right\}$.

One can consider the parameter $a$ as controlling the size of the neighborhood in which the efficient set is declared locally optimal. Usually, $a=1$. For algorithms that allow the user to decide $a$, the tradeoff between small $a$ and large $a$ is one of computational effort. For example, as described in Section 5.1.2, Cooper et al. $(2017,2018)$ perform a neighborhood enumeration step to certify that a candidate-estimated efficient set is (approximately) sample-path locally optimal. The larger the neighborhood, the more computation required for this certification. For large enough $a$, the local efficient set equals the global efficient set. Absent the assumption of structure in the objective functions, declaring a candidate-estimated efficient set as sample-path globally optimal requires obtaining simulation replications from every feasible point.

We consider methods in which the estimated efficient set provably converges to an $\mathcal{N}_{a}$-local efficient set w.p.1 under appropriate regularity conditions. We discuss three such methods in the sections that follow; two are SAA-based methods and one is a non-SAA-based method.

\subsection{SAA-Based Methods for Integer-Ordered MOSO}

We describe two SAA-based methods for integer-ordered MOSO, as follows.

5.1.1 APS. Gutjahr (2009) provides the Adaptive Pareto Search (APS) framework for $d=2$ objectives. Letting $\hat{\mathcal{E}}_{0}\left(n_{0}\right)=\emptyset$, for iteration $v=1,2, \ldots$, APS performs the following steps in iteration $v$ : (1) solution proposal: solve a biobjective Problem $\bar{M}$ for sample size $n_{v}^{(a)}$ yielding the estimated efficient set $\hat{\mathcal{E}}_{v}^{(\mathrm{a})}\left(n_{v}^{(\mathrm{a})}\right)$; (2) solution evaluation: for each $X \in \hat{\mathcal{E}}_{v-1}\left(n_{v-1}\right) \cup \hat{\mathcal{E}}_{v}^{(\mathrm{a})}\left(n_{v}^{(\mathrm{a})}\right)$, independently of Step (1), obtain the estimator $\bar{G}\left(X, n_{v}\right)$; (3) calculate $\hat{\mathcal{E}}_{v}\left(n_{v}\right)$ as the updated estimated efficient set based on the objective function estimates obtained in Step (2). Gutjahr (2009) recommends a constant sample size for $n_{v}^{(a)}$ and a linearly increasing sample size for $n_{v}$. Gutjahr 
(2009) asserts that algorithms implemented in this framework converge under appropriate regularity conditions; whether the convergence is to a local or global efficient set depends on how Step (1) is implemented.

5.1.2 The Epsilon-Constraint Method in a Retrospective Approximation Framework. Cooper et al. $(2017,2018)$ propose an $\varepsilon$-constraint method embedded in an RA framework (see Section 4.3.3) to solve MOSO problems with $d=2$ objectives. The $\varepsilon$-constraint method is a scalarization method that reformulates the MOO problem into a constrained single-objective optimization problem. That is, for a chosen objective $k^{*}$ and vector of constraints $\varepsilon=\left(\varepsilon_{1}, \ldots, \varepsilon_{k^{*}-1}, \varepsilon_{k^{*}+1}, \ldots, \varepsilon_{d}\right)$, the $\varepsilon$-constraint problem is

$$
\text { Problem } S\left(\varepsilon, k^{*}\right): \quad \text { minimize }_{\mathbf{x} \in X} \quad g_{k^{*}}(\mathbf{x}) \quad \text { s.t. } \quad g_{k}(\mathbf{x}) \leq \varepsilon_{k} \text { for all } k=1, \ldots, d ; k \neq k^{*} .
$$

If Problem $S\left(\varepsilon, k^{*}\right)$ is feasible, then its solution is weakly efficient (Miettinen 1999, p. 85, Theorem 3.2.1). Further, a point $\mathrm{x}^{*}$ is efficient if and only if, for all objectives $k^{*}=1, \ldots, d$, it is the solution to Problem $S\left(\varepsilon, k^{*}\right)$ with $\varepsilon_{k}=g_{k}\left(\mathbf{x}^{*}\right)$ for all $k=1, \ldots, d, k \neq k^{*}$ (Miettinen 1999, p. 85, Theorem 3.2.2). The $\varepsilon$-constraint method can retrieve all Pareto-optimal points in nonconvex problems by appropriately varying the $\varepsilon$ vector.

For a given simulation budget $n$ to be spent at each visited point, Cooper et al. (2017) retrieve an estimated efficient set $\hat{\mathcal{E}}(n)$ by solving several sample-path $\varepsilon$-constraint problems,

$$
\text { Problem } \bar{S}\left(\varepsilon, k^{*}, n\right): \quad \text { minimize }_{\mathbf{x} \in X} \quad \bar{G}_{k^{*}}(\mathbf{x}, n) \quad \text { s.t. } \quad \bar{G}_{k}(\mathbf{x}, n) \leq \varepsilon_{k} \text { for } k \in\{1,2\} ; k \neq k^{*},
$$

at a set of carefully chosen $\varepsilon$ values. Each sample-path $\varepsilon$-constraint problem is solved by the SPLINE algorithm (Wang et al. 2013), which chooses a descent direction by constructing pseudo-gradients. To ensure that their estimated efficient set $\hat{\mathcal{E}}(n)$ is a (relaxed) sample-path local efficient set, Cooper et al. (2017) certify $\hat{\mathcal{E}}(n)$ by performing a (relaxed) local neighborhood enumeration step.

To ensure algorithmic efficiency, Cooper et al. (2017) embed their $\varepsilon$-constraint algorithm in an RA framework. Thus, instead of solving a single sample-path biobjective problem at a static sample size $n$ to retrieve an estimated efficient set $\hat{\mathcal{E}}(n)$, they solve a sequence of sample-path problems at increasing sample sizes $n_{v}, v=1,2, \ldots$. The estimated efficient set from the previous RA iteration, $\hat{\mathcal{E}}\left(n_{v-1}\right)$, is used as a warm-start for the $v$ th RA iteration.

The method published in Cooper et al. (2017) can be considered preliminary work on the R$\mathrm{P} \varepsilon \mathrm{RLE}$ algorithm of Cooper et al. (2018), which is a working paper that contains convergence proofs for the methods outlined above. Generally speaking, the R-P $\varepsilon$ RLE algorithm converges under conditions similar to those required for convergence of R-SPLINE (Wang et al. 2013). Cooper et al. (2018) also describe and prove the convergence of R-MINRLE, a naïve benchmark algorithm for integer-ordered MOSO with $d \geq 2$ objectives.

\subsection{Non-SAA Methods for Integer-Ordered MOSO}

To the best of our knowledge, MO-COMPASS is the only non-SAA-based algorithm for integerordered MOSO that is designed to converge to a local efficient set. MO-COMPASS (Li et al. 2015a) is a multiobjective version of the popular COMPASS algorithm for integer-ordered SOSO problems (Hong and Nelson 2006; Xu et al. 2010), and MO-COMPASS provably converges to an $\mathcal{N}_{1}$-local efficient set for $d \geq 2$ objectives.

Like its predecessor COMPASS, MO-COMPASS is an iterative algorithm that maintains a set of desirable solutions called the most promising area (MPA). In each iteration $v$, MO-COMPASS uniformly selects new candidate points from the MPA, applies a simulation allocation rule (SAR) to determine how many samples to obtain from each point visited so far, estimates the objective values of each point, updates the estimated local efficient set, and updates the MPA (see Algorithm 1). 
In the multiobjective context, the $v$ th MPA is defined as the collection of decision points that are closer to the current estimated $\mathcal{N}_{1}$-local efficient set than to any previously visited estimated nonefficient feasible point. Thus, the MPA is adaptive, converging w.p. 1 to an $\mathcal{N}_{1}$-local efficient set as candidate points are evaluated and iterations $v$ increase. Further, MO-COMPASS allows differential sampling across points. MO-COMPASS may be terminated when a prespecified simulation budget is exhausted or some other criteria are met.

Like COMPASS, versions of MO-COMPASS exist for the case of a fully constrained, partially constrained, or unconstrained feasible region $\mathcal{X}$. For simplicity, we consider only the fully constrained MO-COMPASS algorithm in Algorithm 1; we refer the reader to Li et al. (2015a) for the other cases.

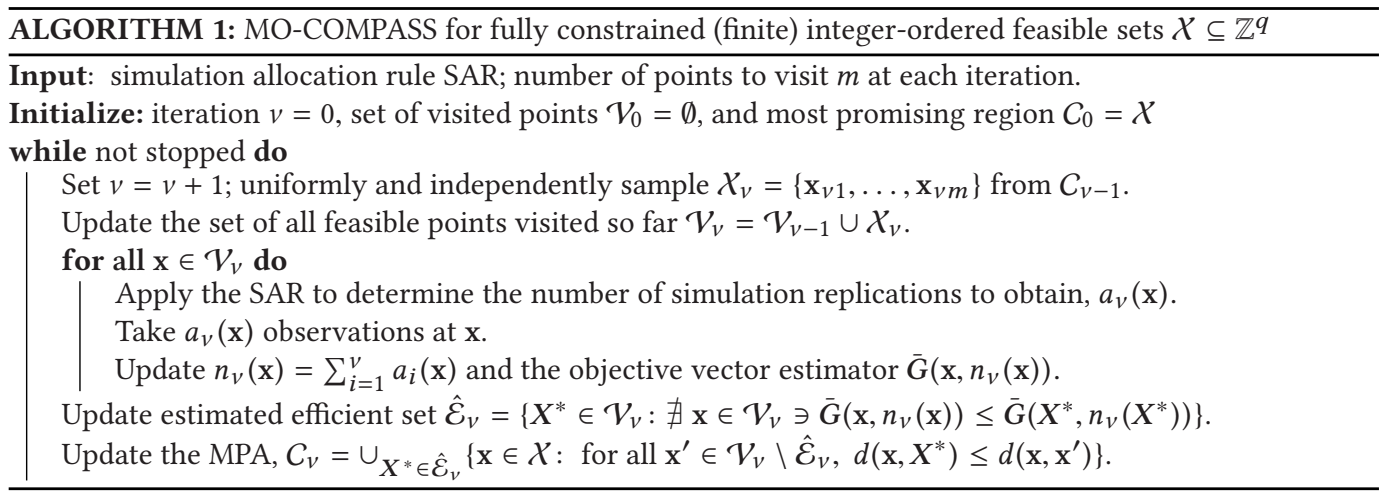

The estimated efficient set $\hat{\mathcal{E}}_{v}$ constructed by the fully constrained MO-COMPASS algorithm converges to an $\mathcal{N}_{1}$-local efficient set w.p.1. This convergence holds when

(1) no two points have exactly the same performance on any objective-that is, there exists $\delta>0$ such that for all $\mathbf{x}, \mathbf{x}^{\prime} \in \mathcal{X}$, and for all $k \in\{1, \ldots, d\}, \mathbf{x} \neq \mathbf{x}^{\prime}$ implies $\left|g_{k}(\mathbf{x})-g_{k}\left(\mathbf{x}^{\prime}\right)\right| \geq \delta$

(2) for every $\mathbf{x} \in \mathcal{X}$ and objective $k \in\{1, \ldots, d\}, \bar{G}_{k}(\mathbf{x}, n) \rightarrow g_{k}(\mathbf{x})$ w.p. 1 as $n \rightarrow \infty$; and

(3) the SAR guarantees that at least one simulation replication is obtained at all newly sampled points in each iteration, that is, $a_{v}(\mathbf{x}) \geq 1$ if $\mathbf{x}$ is a newly visited point at iteration $v$; and each visited point is sampled infinitely often, so that $\lim _{v \rightarrow \infty} n_{v}(\mathbf{x})=\infty$ for all $\mathrm{x} \in \cup_{v=0}^{\infty} \mathcal{V}_{v}$.

Note that MO-COMPASS allows the use of CRN. The convergence of MO-COMPASS for the partially constrained and unconstrained cases requires additional conditions.

\section{TESTBEDS AND SOFTWARE}

To the best of our knowledge, no testbeds and few software resources exist for MOSO. The opensource PyMOSO package (Cooper and Hunter 2018) provides off-the-shelf implementations of the $\mathrm{R}-\mathrm{P} \varepsilon \mathrm{RLE}$ and R-MinRLE algorithms for integer-ordered MOSO (see Section 5.1.2). Also, code for many algorithms usually can be obtained by emailing the authors. The simopt.org problem and solver library currently contains freely available SOSO test problems and software; in the future, we anticipate that it will contain MOSO test problems and software (Henderson and Pasupathy 2018). Likewise, the International Society on MCDM maintains a website with software tools at https://www.mcdmsociety.org. 
To create a MOSO testbed, one may begin by studying the many well-developed MOO testbeds, since noise may be added to these problems. MOO problem testbeds include problems with a variety of features. According to Deb et al. (2002), a good MOO test problem should (1) be easy to construct, (2) be scalable in both the number of decision variables and number of objective functions, (3) have a Pareto set whose location and shape are known exactly, and (4) have structural properties that make convergence to the efficient set and the even distribution of Pareto points nontrivial. Existing testbeds, such as those in Deb (1999), Deb et al. (2001, 2002), Huband et al. (2006), and Zitzler et al. (2000), include scalable problems with nonconvex objective functions, multiple local efficient sets, and disconnected Pareto sets. Allmendinger et al. (2016, pp. 15-17) provide a table of commonly used test problems, and Cheng et al. (2017) provide a recent critique of existing testbeds and suggest a set of benchmark problems. We refer the reader to Cheng et al. (2017) for a discussion of test problem constructions and references to additional MOO problem testbeds.

\section{OPEN QUESTIONS AND CONCLUDING REMARKS}

As may be apparent from our discussion of existing MOSO theory, methods, and algorithms, a tremendous amount of work remains in this area. We make the following general remarks about the future development of MOSO theory, methods, and algorithms.

(1) We believe that all future MOSO methods should be developed with parallel algorithmic implementation in mind. To solve increasingly large and difficult MOSO problems, we must fully exploit the capabilities of now-ubiquitous parallel computing platforms, that is, computing platforms in which multiple processors can independently execute code and communicate by accessing shared memory or with message passing.

(2) Because of their special structure, MOSO problems with exactly two objectives often are easier to solve than problems with three or more objectives. Thus, developing good solution methods for biobjective problems is an important and impactful (see Section 1.1) first step to developing comprehensive MOSO solution methods.

We now remark on four specific areas for future development. The first two areas are inspired by the MOO literature, and the rest are inspired by the SOSO literature.

Scalarization Methods. While we discuss the use of scalarization by, e.g., Bonnel and Collonge (2015), Cooper et al. (2017), Fliege and Xu (2011), and Kim and Ryu (2011a), additional theoretical, methodological, and algorithmic development is required for common MOO scalarization techniques in a MOSO context. As a notable example, the U.S. Department of Energy is using the $\varepsilon$-constraint method to solve MOSO problems in the context of the Advanced Process Engineering Co-Simulator (Subramanyan et al. 2011). To the best of our knowledge, apart from Cooper et al. $(2017,2018)$ and the existing literature on stochastically constrained SOSO methods, there is no theoretical or methodological development for this scalarization technique in the MOSO context. Further, many scalarization methods exist beyond those we discuss; see, e.g., Das and Dennis (1998), Eichfelder (2008), Messac et al. (2003), and Pascoletti and Serafini (1984). Additional development of theory, methods, and algorithms for scalarization in MOSO may help employ the full power of existing SOSO algorithms toward solving MOSO problems.

Adaptive Methods for an Evenly Spaced Pareto Representation. Ideally, the points returned by a MOSO solution method should be sufficiently spread out in the Pareto set when the algorithm terminates, and not clustered together in one region. Because MOSO problems may be more computationally intensive than MOO problems, adaptive methods to find evenly distributed points in the Pareto set are likely to be crucial for solving MOSO problems efficiently-such methods will 
not waste effort obtaining high-precision estimates of many points clustered together in one region of the Pareto set while failing to explore other regions. To this end, MOSO researchers may find adaptive algorithms from the MOO context helpful, including the adaptive scalarization methods of Eichfelder (2008), the derivative pursuit method of Singhee and Castalino (2010), and the adaptive weighting scheme of Deshpande et al. (2016).

Fixed-Precision MORS Procedures. As noted in Section 3.2, there is a general lack of development in fixed-precision MORS procedures. Given the importance, prominence, and popularity of fixedprecision single-objective $R \& S$ procedures and the recent advances on single-objective parallel R\&S procedures (Hunter and Nelson 2017), we believe developing analogous MORS procedures would be an important advance. Work on the multivariate Behrens-Fisher problem and the Heteroscedastic Method (see, e.g., Anderson 2003; Dudewicz et al. 1991; Dudewicz and Taneja 1987) may be relevant to designing such procedures.

Adaptive Sampling. Adaptive sampling methods are among the most efficient methods available for SOSO (Bottou et al. 2017; Hashemi et al. 2017; Pasupathy et al. 2018; Pasupathy and Schmeiser 2010; Shashaani et al. 2018). Given the difficulty of MOSO problems, adaptive sampling methods may be especially useful when designing MOSO algorithms-the ideal algorithm for continuous feasible sets perhaps being some form of adaptive sampling algorithm that also employs adaptive methods for an evenly spaced Pareto representation. For entry points to the adaptive sampling literature, see Bottou et al. (2017).

\section{ACKNOWLEDGMENTS}

This article began in the special topics class IE 690 Simulation Optimization at Purdue University in Fall 2016. The authors thank class members Zibo Zhao and Zhouyang Lou for participating in draft papers created during the course. The authors also thank the anonymous referees for comments that improved the article; David Eckman, Raghu Pasupathy, and Mario Ventresca for commenting on early versions of the current article; Mickaël Binois for pointing us to the kriging literature; and Kalyani Nagaraj and Raghu Pasupathy for interesting discussions related to MOSO in general.

\section{REFERENCES}

F. B. Abdelaziz. 2012. Solution approaches for the multiobjective stochastic programming. European fournal of Operational Research 216 (2012), 1-16. DOI : https://doi.org/10.1016/j.ejor.2011.03.033

M. Akan, O. Alagoz, B. Ata, F. S. Erenay, and A. Said. 2012. A broader view of designing the liver allocation system. Operations Research 60, 4 (2012), 757-770. DOI : https://doi.org/10.1287/opre.1120.1064

R. Allmendinger, M. T. M. Emmerich, J. Hakanen, Y. Jin, and E. Rigoni. 2016. Surrogate-assisted multicriteria optimization: Complexities, prospective solutions, and business case. Journal of Multi-Criteria Decision Analysis 24, 1-2 (JanuaryApril 2016), 5-24. DOI : https://doi.org/10.1002/mcda.1605

M. H. Alrefaei and A. H. Diabat. 2009. A simulated annealing technique for multi-objective simulation optimization. Applied Mathematics and Computation 215, 8 (2009), 3029-3035. DOI : https://doi.org/10.1016/j.amc.2009.09.051

S. Amaran, N. V. Sahinidis, B. Sharda, and S. J. Bury. 2016. Simulation optimization: A review of algorithms and applications. Annals of Operations Research 240, 1 (May 2016), 351-380. DOI : https://doi.org/10.1007/s10479-015-2019-x

L. Amodeo, C. Prins, and D. R. Sánchez. 2009. Comparison of metaheuristic approaches for multi-objective simulationbased optimization in supply chain inventory management. In Applications of Evolutionary Computing, M. Giacobini, A. Brabazon, S. Cagnoni, G. A. Di Caro, A. Ekárt, A. I. Esparcia-Alcázar, M. Farooq, A. Fink, P. Machado, J. McCormack, F. Neri, M. O’Neill, M. Preuss, F. Rothlauf, E. Tarantino, and S. Yang (Eds.). Springer, Berlin, 798-807.

T. W. Anderson. 2003. An Introduction to Multivariate Statistical Analysis (3rd ed.). Wiley-Interscience, Hoboken, NJ.

M. Andersson, H. Grimm, A. Persson, and A. Ng. 2007. A web-based simulation optimization system for industrial scheduling. In Proceedings of the 2007 Winter Simulation Conference, S. G. Henderson, B. Biller, M.-H. Hsieh, J. Shortle, J. D. Tew, and R. R. Barton (Eds.). IEEE, Piscataway, NJ, 1844-1852. 
S. Andradóttir. 2015. A review of random search methods. In Handbook of Simulation Optimization, M. C. Fu (Ed.). International Series in Operations Research \& Management Science, Vol. 216. Springer, New York, 277-292. DOI : https: //doi.org/10.1007/978-1-4939-1384-8_10

E. A. Applegate, G. Feldman, S. R. Hunter, and R. Pasupathy. 2018. Multi-objective ranking and selection: Optimal sampling laws and tractable approximations via SCORE. Optimization Online (2018). Retrieved from http://www. optimization-online.org/DB_HTML/2018/07/6750.html.

C. Audet, J. Bigeon, D. Cartier, S. Le Digabel, and L. Salomon. 2018. Performance indicators in multiobjective optimization. Optimization Online (2018). Retrieved from http://www.optimization-online.org/DB_HTML/2018/10/6887.html.

C. Audet, G. Savard, and W. Zghal. 2008. Multiobjective optimization through a series of single-objective formulations. SIAM fournal on Optimization 19, 1 (2008), 188-210. DOI : https://doi.org/10.1137/060677513

F. F. Baesler and J. A. Sepulveda. 2000. Simulation optimization: Multi-response simulation optimization using stochastic genetic search within a goal programming framework. In Proceedings of the 2000 Winter Simulation Conference, J. A. Joines, R. R. Barton, K. Kang, and P. A. Fishwick (Eds.). IEEE, Piscataway, NJ, 788-794.

F. F. Baesler and J. A. Sepulveda. 2001. Multi-objective simulation optimization for a cancer treatment center. In Proceedings of the 2001 Winter Simulation Conference, B. A. Peters, J. S. Smith, D. J. Medeiros, and M. W. Rohrer (Eds.). IEEE, Piscataway, NJ, 1405-1411.

D. Batur and F. F. Choobineh. 2010. Mean-variance based ranking and selection. In Proceedings of the 2010 Winter Simulation Conference, B. Johansson, S. Jain, J. Montoya-Torres, J. Hugan, and E. Yücesan (Eds.). IEEE, Piscataway, NJ.

D. Bertsimas, V. F. Farias, and N. Trichakis. 2013. Fairness, efficiency, and flexibility in organ allocation for kidney transplantation. Operations Research 61, 1 (2013), 73-87. DOI : https://doi.org/10.1287/opre.1120.1138

L. Bianchi, M. Dorigo, L. M. Gambardella, and W. J. Gutjahr. 2009. A survey on metaheuristics for stochastic combinatorial optimizaiton. Natural Computing 8 (2009), 239-287. DOI : https://doi.org/10.1007/s11047-008-9098-4

P. Billingsley. 1995. Probability and Measure (3rd ed.). John Wiley and Sons, New York.

J. Boesel, B. L. Nelson, and S.-H. Kim. 2003. Using ranking and selection to "clean up" after simulation optimization. Operations Research 51, 5 (2003), 814-825.

H. Bonnel and C. Collonge. 2014. Stochastic optimization over a Pareto set associated with a stochastic multi-objective optimization problem. fournal of Optimization Theory and Applications 162 (2014), 405-427. DOI : https://doi.org/10.1007/ s10957-013-0367-8

H. Bonnel and C. Collonge. 2015. Optimization over the Pareto outcome set associated with a convex bi-objective optimization problem: Theoretical results, deterministic algorithm and application to the stochastic case. Fournal of Global Optimization 62 (2015), 481-505. DOI : https://doi.org/10.1007/s10898-014-0257-0

L. Bottou, F. E. Curtis, and J. Nocedal. 2017. Optimization methods for large-scale machine learning. ArXiv e-prints (June 2017), 95. arXiv:1606.04838. Retrieved from https://arxiv.org/abs/1606.04838

J. Branke and W. Zhang. 2015. A new myopic sequential sampling algorithm for multi-objective problems. In Proceedings of the 2015 Winter Simulation Conference, L. Yilmaz, W. K. V. Chan, I. Moon, T. M. K. Roeder, C. Macal, and M. D. Rossetti (Eds.). IEEE, Piscataway, NJ, 3589-3598.

J. Branke, W. Zhang, and Y. Tao. 2016. Multiobjective ranking and selection based on hypervolume. In Proceedings of the 2016 Winter Simulation Conference, T. M. K. Roeder, P. I. Frazier, R. Szechtman, E. Zhou, T. Huschka, and S. E. Chick (Eds.). IEEE, Piscataway, NJ, 859-870.

D. Brockhoff. 2011. Theoretical aspects of evolutionary multiobjective optimization. In Theory of Randomized Search Heuristics: Foundations and Recent Developments, A. Auger and B. Doerr (Eds.). Series on Theoretical Computer Science, Vol. 1. World Scientific, Hackensack, NJ, 101-139. DOI : https://doi.org/10.1142/9789814282673_0004

D. Büche, P. Stoll, R. Dornberger, and P. Koumoutsakos. 2002. Multiobjective evolutionary algorithm for the optimization of noisy combustion processes. IEEE Transactions on Systems, Man, and Cybernetics - Part C: Applications and Reviews 32, 4 (2002), 460-473.

J. C. Butler, D. J. Morrice, and P. Mullarkey. 2001. A multiple attribute utility theory approach to ranking and selection. Management Science 47, 6 (2001), 800-816.

G. Casella and R. L. Berger. 2002. Statistical Inference (2nd ed.). Duxbury, Pacific Grove, CA.

T. M. Chan. 2013. Klee's measure problem made easy. In 2013 IEEE 54th Annual Symposium on Foundations of Computer Science. IEEE, Piscataway, NJ, 410-419. DOI : https://doi.org/10.1109/FOCS.2013.51

W. K. V. Chan, A. D’Ambrogio, G. Zacharewicz, N. Mustafee, G. Wainer, and E. Page (Eds.). 2017. Proceedings of the 2017 Winter Simulation Conference. IEEE, Piscataway, NJ. Retrieved from http://meetings2.informs.org/wordpress/wsc2017/ tracks/.

V. Chankong and Y. Y. Haimes. 1983. Multiobjective Decision Making: Theory and Methodology. Elsevier Science Publishing Co., New York.

C.-H. Chen, Q.-S. Jia, and L. H. Lee (Eds.). 2013. Stochastic Simulation Optimization for Discrete Event Systems: Perturbation Analysis, Ordinal Optimization, and Beyond. World Scientific, Singapore. 
C.-H. Chen and L. H. Lee. 2010. Stochastic Simulation Optimization: An Optimal Computing Budget Allocation. World Scientific, Hackensack, NJ.

C.-H. Chen, J. Lin, E. Yücesan, and S. E. Chick. 2000. Simulation budget allocation for further enhancing the efficiency of ordinal optimization. Discrete Event Dynamic Systems 10, 3 (2000), 251-270. DOI : https://doi.org/10.1023/A:1008349927281

E. J. Chen and W. D. Kelton. 2005. Sequential selection procedures: Using sample means to improve efficiency. European fournal of Operational Research 166, 1 (2005), 133-153. DOI : https://doi.org/10.1016/j.ejor.2003.01.003

E. J. Chen and L. H. Lee. 2009. A multi-objective selection procedure of determining a Pareto set. Computers \& Operations Research 36 (2009), 1872-1879. DOI : https://doi.org/10.1016/j.cor.2008.06.003

T.-L. Chen and C.-C. Wang. 2016. Multi-objective simulation optimization for medical capacity allocation in emergency department. Journal of Simulation 10, 1 (2016), 50-68. DOI : https://doi.org/10.1057/jos.2014.39

R. Cheng, M. Li, Y. Tian, X. Zhang, S. Yang, Y. Jin, and X. Yao. 2017. A benchmark test suite for evolutionary many-objective optimization. Complex \& Intelligent Systems 3, 1 (2017), 67-81. DOI : https://doi.org/10.1007/s40747-017-0039-7

E. P. Chew, L. H. Lee, S. Teng, and C. H. Koh. 2009. Differentiated service inventory optimization using nested partitions and MOCBA. Computers \& Operations Research 36, 5 (2009), 1703-1710. DOI : https://doi.org/10.1016/j.cor.2008.04.006

M. Chica, A. A. Juan Pérez, O. Cordon, and D. Kelton. 2017. Why simheuristics? Benefits, limitations, and best practices when combining metaheuristics with simulation. SSRN (February 2017). DOI : https://doi.org/10.2139/ssrn.2919208

S. E. Chick, J. Branke, and C. Schmidt. 2010. Sequential sampling to myopically maximize the expected value of information. INFORMS fournal on Computing 22, 1 (2010), 71-80.

A. Chinchuluun and P. M. Pardalos. 2007. A survey of recent developments in multiobjective optimization. Annals of Operations Research 154 (2007), 29-50.

S. H. Choi and T. G. Kim. 2018. Pareto set selection for multiobjective stochastic simulation model. IEEE Transactions on Systems, Man, and Cybernetics: Systems (2018). DOI : https://doi.org/10.1109/TSMC.2018.2846680

C. A. Coello Coello, G. B. Lamont, and D. A. van Veldhuizen. 2007. Evolutionary Algorithms for Solving Multi-Objective Problems. Springer US, Boston, MA. 800 pages.

K. Cooper and S. R. Hunter. 2018. PyMOSO: Software for multi-objective simulation optimization with R-PERLE and R-MinRLE. Optimization Online (2018). Retrieved from http://www.optimization-online.org/DB_HTML/2018/10/6876 https://github.com/HunterResearch/PyMOSO.

K. Cooper, S. R. Hunter, and K. Nagaraj. 2017. An epsilon-constraint method for integer-ordered bi-objective simulation optimization. In Proceedings of the 2017 Winter Simulation Conference, W. K. V. Chan, A. D'Ambrogio, G. Zacharewicz, N. Mustafee, G. Wainer, and E. Page (Eds.). IEEE, Piscataway, NJ, 2303-2314. DOI : https://doi.org/10.1109/WSC.2017. 8247961

K. Cooper, S. R. Hunter, and K. Nagaraj. 2018. Bi-objective simulation optimization on integer lattices using the epsilonconstraint method in a retrospective approximation framework. Optimization Online (2018). Retrieved from http://www. optimization-online.org/DB_HTML/2018/06/6649.html.

O. Crespo, J. E. Bergez, and F. Garcia. 2010. Multiobjective optimization subject to uncertainty: Application to irrigation strategy management. Computers and Electronics in Agriculture 74 (2010), 145-154. DOI: https://doi.org/10.1016/ j.compag.2010.07.009

I. Das and J. E. Dennis. 1997. A closer look at drawbacks of minimizing weighted sums of objectives for Pareto set generation in multicriteria optimization problems. Structural Optimization 14, 1 (1997), 63-69.

I. Das and J. E. Dennis. 1998. Normal-boundary intersection: A new method for generating the Pareto surface in nonlinear multicriteria optimization problems. SIAM fournal on Optimization 8, 3 (1998), 631-657.

K. Deb. 1999. Multi-objective genetic algorithms: Problem difficulties and construction of test problems. Evolutionary Computation 7, 3 (1999), 205-230.

K. Deb. 2009. Multi-objective Optimization Using Evolutionary Algorithms. Wiley, Hoboken, NJ.

K. Deb, L. Thiele, M. Laumanns, and E. Zitzler. 2001. Scalable Test Problems for Evolutionary Multi-objective Optimization. Technical Report. Institut für Technische Informatik und Kommunikationsnetze, ETH Zürich. TIK-Technical Report No. 112 .

K. Deb, L. Thiele, M. Laumanns, and E. Zitzler. 2002. Scalable multi-objective optimization test problems. In Proceedings of the 2002 Congress on Evolutionary Computation, Vol. 1. IEEE, Piscataway, NJ, 825-830. DOI : https://doi.org/10.1109/CEC. 2002.1007032

S. Deshpande, L. T. Watson, and R. A. Canfield. 2016. Multiobjective optimization using an adaptive weighting scheme. Optimization Methods and Software 31, 1 (2016), 110-133. DOI : https://doi.org/10.1080/10556788.2015.1048861

J. A. Désidéri. 2012. Multiple-gradient descent algorithm (MGDA) for multiobjective optimization. Comptes Rendus Mathematique 350, 5-6 (2012), 313-318. DOI : https://doi.org/10.1016/j.crma.2012.03.014

H. Ding, L. Benyoucef, and X. Xie. 2006. A simulation-based multi-objective genetic algorithm approach for networked enterprises optimization. Engineering Applications of Artificial Intelligence 19 (2006), 609-623. DOI : https://doi.org/10. 1016/j.engappai.2005.12.008 
M. Drugan and B. Manderick. 2015. Exploration versus exploitation trade-off in infinite horizon Pareto Multi-Armed bandits algorithms. In Proceedings of the International Conference on Agents and Artificial Intelligence (ICAART'15). SCITEPRESS, Portugal, 66-77. DOI : https://doi.org/10.5220/0005195500660077

E. J. Dudewicz, H. Hyakutake, and V. S. Taneja. 1991. The heteroscedastic method II: Simplified sampling. American fournal of Mathematical and Management Sciences 11, 3-4 (1991), 263-276. DOI : https://doi.org/10.1080/01966324.1991.10737311

E. J. Dudewicz and V. S. Taneja. 1978. Multivariate ranking and selection without reduction to a univariate problem. In Proceedings of the 1978 Winter Simulation Conference, H. J. Highland, N. R. Nielsen, and L. G. Hull (Eds.). IEEE, Piscataway, NJ, 207-210.

E. J. Dudewicz and V. S. Taneja. 1981. A multivariate solution of the multivariate ranking and selection problem. Communications in Statistics - Theory and Methods 10, 18 (1981), 1849-1868. DOI : https://doi.org/10.1080/03610928108828154

E. J. Dudewicz and V. S. Taneja. 1987. The heteroscedastic method: Multivariate implementation. Annals of the Institute of Statistical Mathematics 39 (1987), 177-190. DOI : https://doi.org/10.1007/BF02491458

C. Dullinger, W. Struckl, and M. Kozek. 2017. Simulation-based multi-objective system optimization of train traction systems. Simulation Modelling Practice and Theory 72 (2017), 104-117. DOI : https://doi.org/10.1016/j.simpat.2016.12.008

M. Ehrgott. 2005. Multicriteria Optimization (2nd ed.). Lecture Notes in Economics and Mathematical Systems, Vol. 491. Springer, Heidelberg.

M. Ehrgott. 2012. Vilfredo Pareto and multi-objective optimization. In Optimization Stories: 21st International Symposium on Mathematical Programming, M. Grötschel (Ed.). Documenta mathematica, Bielefeld, Germany, 447-453.

M. Ehrgott, X. Gandibleux, and A. Przybylski. 2016. Exact methods for multi-objective combinatorial optimisation. In Multiple Criteria Decision Analysis: State of the Art Surveys, S. Greco, M. Ehrgott, and J. R. Figueira (Eds.). International Series in Operations Research \& Management Science, Vol. 233. Springer, New York, 817-850. DOI : https: //doi.org/10.1007/978-1-4939-3094-4_19

M. Ehrgott and D. Tenfelde-Podehl. 2003. Computation of ideal and nadir values and implications for their use in MCDM methods. European fournal of Operational Research 151 (2003), 119-139.

M. Ehrgott and M. M. Wiecek. 2005. Multiobjective programming. In Multiple Criteria Decision Analysis: State of the Art Surveys, J. Figueira, S. Greco, and M. Ehrgott (Eds.). International Series in Operations Research \& Management Science, Vol. 78. Springer, New York. DOI: https://doi.org/10.1007/0-387-23081-5_17

G. Eichfelder. 2008. Adaptive Scalarization Methods in Multiobjective Optimization. Springer, Berlin.

H. Eskandari and G. D. Geiger. 2009. Evolutionary multiobjective optimization in noisy problem environments. fournal on Heuristics 15 (2009), 559-595. DOI : https://doi.org/10.1007/s10732-008-9077-z

G. W. Evans, B. Stuckman, and M. Mollaghasemi. 1991. Multicriteria optimization of simulation models. In Proceedings of the 1991 Winter Simulation Conference, B. L. Nelson, W. D. Kelton, and G. M. Clark (Eds.). IEEE, Piscataway, NJ, 894-900. DOI : https://doi.org/10.1109/WSC.1991.185702

S. L. Faulkenberg and M. M. Wiecek. 2010. On the quality of discrete representations in multiple objective programming. Optimization and Engineering 11 (2010), 423-440. DOI : https://doi.org/10.1007/s11081-009-9099-x

G. Feldman. 2017. Sampling Laws for Multi-objective Simulation Optimization on Finite Sets. Ph.D. Dissertation. Purdue University.

G. Feldman and S. R. Hunter. 2018. SCORE allocations for bi-objective ranking and selection. ACM Transactions on Modeling and Computer Simulation 28, 1, Article 7 (January 2018), 28 pages. DOI : https://doi.org/10.1145/3158666

G. Feldman, S. R. Hunter, and R. Pasupathy. 2015. Multi-objective simulation optimization on finite sets: Optimal allocation via scalarization. In Proceedings of the 2015 Winter Simulation Conference, L. Yilmaz, W. K. V. Chan, I. Moon, T. M. K. Roeder, C. Macal, and M. D. Rossetti (Eds.). IEEE, Piscataway, NJ, 3610-3621. DOI : https://doi.org/10.1109/WSC.2015. 7408520

W. H. Feng, N. Kong, and H. Wan. 2013. A simulation study of cadaveric liver allocation with a single-score patient prioritization formula. Journal of Simulation 7, 2 (2013), 109-125.

J. Fliege, L. M. Graña Drummond, and B. F. Svaiter. 2009. Newton's method for multiobjective optimization. SIAM fournal on Optimization 20, 2 (2009), 602-626. DOI : https://doi.org/10.1137/08071692X

J. Fliege and B. F. Svaiter. 2000. Steepest descent methods for multicriteria optimization. Mathematical Methods of Operations Research 51, 3 (2000), 479-494. DOI : https://doi.org/10.1007/s001860000043

J. Fliege and R. Werner. 2014. Robust multiobjective optimization \& applications in portfolio optimization. European fournal of Operational Research 234 (2014), 422-433. DOI : https://doi.org/10.1016/j.ejor.2013.10.028

J. Fliege and H. Xu. 2011. Stochastic multiobjective optimization: Sample average approximation and applications. fournal of Optimization Theory and Applications 151 (2011), 135-162. DOI : https://doi.org/10.1007/s10957-011-9859-6

P. I. Frazier and A. M. Kazachkov. 2011. Guessing preferences: A new approach to multi-attribute ranking and selection. In Proceedings of the 2011 Winter Simulation Conference, S. Jain, R. R. Creasey, J. Himmelspach, K. P. White, and M. Fu (Eds.). IEEE, Piscataway, NJ, 4324-4336. 
L. W. Friedman. 1987. Systems simulation. Design and analysis of multivariate response simulations: The state of the art. Behavioral Science 32 (1987), 138-148.

M. Fu. 1994. Optimization via simulation: A review. Annals of Operations Research 53 (1994), 199-247.

M. C. Fu. 2002. Optimization for simulation: Theory vs. practice. INFORMS fournal on Computing 14 (2002), 192-215. DOI : https://doi.org/10.1287/ijoc.14.3.192.113

M. C. Fu (Ed.). 2015. Handbook of Simulation Optimization. International Series in Operations Research \& Management Science, Vol. 216. Springer, New York. DOI : https://doi.org/10.1007/978-1-4939-1384-8

M. C. Fu, G. Bayraksan, S. G. Henderson, B. L. Nelson, W. B. Powell, I. O. Ryzhov, and B. Thengvall. 2014. Simulation optimization: A panel on the state of the art in research and practice. In Proceedings of the 2014 Winter Simulation Conference, A. Tolk, S. Y. Diallo, I. O. Ryzhov, L. Yilmaz, S. Buckley, and J. A. Miller (Eds.). IEEE, Piscataway, NJ, 36963706. DOI : https://doi.org/10.1109/WSC.2014.7020198

D. Goldsman. 2015. A practical guide to ranking and selection methods. In TutORials in Operations Research, D. M. Aleman and A. C. Thiele (Eds.). INFORMS, Catonsville, MD, Chapter 6, 89-110. DOI : https://doi.org/10.1287/educ.2015.0140

S. Gören, A. Baccouche, and H. Pierreval. 2017. A framework to incorporate decision-maker preferences into simulation optimization to support collaborative design. IEEE Transactions on Systems, Man, and Cybernetics: Systems 47, 2 (February 2017), 229-237. DOI : https://doi.org/10.1109/TSMC.2016.2531643

W. J. Gutjahr. 2005. Two metaheuristics for multiobjective stochastic combinatorial optimization. In Stochastic Algorithms: Foundations and Applications: 3rd International Symposium (SAGA'05), O. B. Lupanov, O. M. Kasim-Zade, A. V. Chaskin, and K. Steinhöfel (Eds.). Springer-Verlag, Berlin, 116-125.

W. J. Gutjahr. 2009. A provably convergent heuristic for stochastic bicriteria integer programming. Fournal on Heuristics 15 (2009), 227-258. DOI : https://doi.org/10.1007/s10732-008-9071-5

W. J. Gutjahr. 2011. Recent trends in metaheuristics for stochastic combinatorial optimization. Central European fournal of Computer Science 1, 1 (2011), 58-66. DOI : https://doi.org/10.2478/s13537-011-0003-3

W. J. Gutjahr and A. Pichler. 2016. Stochastic multi-objective optimization: A survey on non-scalarizing methods. Annals of Operations Research 236, 2 (January 2016), 475-499. DOI : https://doi.org/10.1007/s10479-013-1369-5

F. S. Hashemi, R. Pasupathy, and M. R. Taaffe. 2017. The adaptive sampling gradient method: Optimizing smooth functions with an inexact oracle. Optimization Online (May 2017). Retrieved from http://www.optimization-online.org/ DB_HTML/2017/05/6045.html.

S. G. Henderson and R. Pasupathy. 2018. Simulation Optimization Library. Retrieved from http://www.simopt.org.

S. Hoffenson, S. Arepally, and P. Y. Papalambros. 2014. A multi-objective optimization framework for assessing military ground vehicle design for safety. fournal of Defense Modeling and Simulation: Applications, Methodology, Technology 11, 1 (2014), 33-46. DOI : https://doi.org/10.1177/1548512912459596

T. Homem-de-Mello and G. Bayraksan. 2015. Stochastic constraints and variance reduction techniques. In Handbook of Simulation Optimization, M. C. Fu (Ed.). International Series in Operations Research \& Management Science, Vol. 216. Springer, New York, 245-276. DOI : https://doi.org/10.1007/978-1-4939-1384-8_9

L. J. Hong and B. L. Nelson. 2006. Discrete optimization via simulation using COMPASS. Operations Research 54, 1 (JanuaryFebruary 2006), 115-129. DOI : https://doi.org/10.1287/opre.1050.0237

L. J. Hong and B. L. Nelson. 2009. A brief introduction to optimization via simulation. In Proceedings of the 2009 Winter Simulation Conference, M. D. Rossetti, R. R. Hill, B. Johansson, A. Dunkin, and R. G. Ingalls (Eds.). IEEE, Piscataway, NJ, 75-85. DOI : https://doi.org/10.1109/WSC.2009.5429321

L. J. Hong, B. L. Nelson, and J. Xu. 2015. Discrete optimization via simulation. In Handbook of Simulation Optimization, M. C. Fu (Ed.). International Series in Operations Research \& Management Science, Vol. 216. Springer, New York, 9-44. DOI : https://doi.org/10.1007/978-1-4939-1384-8_1

H. Huang. 2016. Discrete-Event Simulation and Optimization to Improve the Performance of a Healthcare System. Ph.D. Dissertation. University of Washington.

H. Huang and Z. B. Zabinsky. 2014. Multiple objective probabilistic branch and bound for Pareto optimal approximation. In Proceedings of the 2014 Winter Simulation Conference, A. Tolk, S. Y. Diallo, I. O. Ryzhov, L. Yilmaz, S. Buckley, and J. A. Miller (Eds.). IEEE, Piscataway, NJ, 3916-3927. DOI : https://doi.org/10.1109/WSC.2014.7020217

S. Huband, P. Hingston, L. Barone, and L. While. 2006. A review of multiobjective test problems and a scalable test problem toolkit. IEEE Transactions on Evolutionary Computation 10, 5 (2006), 477-506. DOI : https://doi.org/10.1109/TEVC.2005. 861417

S. R. Hunter and B. McClosky. 2016. Maximizing quantitative traits in the mating design problem via simulation-based Pareto estimation. IIE Transactions 48, 6 (2016), 565-578. DOI : https://doi.org/10.1080/0740817X.2015.1096430

S. R. Hunter and B. L. Nelson. 2017. Parallel ranking and selection. In Advances in Modeling and Simulation: Seminal Research from 50 Years of Winter Simulation Conferences, A. Tolk, J. Fowler, G. Shao, and E. Yücesan (Eds.). Springer International, Switzerland, Chapter 12, 249-275. DOI : https://doi.org/10.1007/978-3-319-64182-9 
C.-L. Hwang and A. S. M. Masud. 1979. Multiple Objective Decision Making - Methods and Applications: A State-of-the-Art Survey. Springer-Verlag, Berlin.

S. H. Jacobson and L. W. Schruben. 1989. Techniques for simulation response optimization. Operations Research Letters 8 (February 1989), 1-9. DOI : https://doi.org/10.1016/0167-6377(89)90025-4

J. Jahn. 2011. Vector Optimization: Theory, Applications, and Extensions (2 ed.). Springer-Verlag, Berlin.

N. Jian and S. G. Henderson. 2015. An introduction to simulation optimization. In Proceedings of the 2015 Winter Simulation Conference, L. Yilmaz, W. K. V. Chan, T. M. K. Roeder, C. Macal, and M.D. Rosetti (Eds.). IEEE, Piscataway, NJ, 1780-1794.

S. Jiang, Y.-S. Ong, J. Zhang, and L. Feng. 2014. Consistencies and contradictions of performance metrics in multiobjective optimization. IEEE Transactions on Cybernetics 44, 12 (2014), 2391-2404. DOI : https://doi.org/10.1109/TCYB.2014.2307319

Y. Jin and J. Branke. 2005. Evolutionary optimization in uncertain environments: A survey. IEEE Transactions on Evolutionary Computation 7, 3 (June 2005), 303-317. DOI : https://doi.org/10.1109/TEVC.2005.846356

J. A. Joines, D. Gupta, M. A. Gokce, R. E. King, and M. G. Kay. 2002. Supply chain multi-objective simulation optimization. In Proceedings of the 2002 Winter Simulation Conference, E. Yücesan, C. H. Chen, J. L. Snowdon, and J. M. Charnes (Eds.) IEEE, Piscataway, NJ, 1306-1314. DOI : https://doi.org/10.1109/WSC.2002.1166395

S. Kim. 2014. A derivative-free trust-region method for biobjective optimization. Retrieved from https://vimeo.com/ 83787562.

S. Kim, R. Pasupathy, and S. G. Henderson. 2015. A guide to sample average approximation. In Handbook of Simulation Optimization, M. Fu (Ed.). International Series in Operations Research \& Management Science, Vol. 216. Springer-Verlag, New York, 207-243. DOI: https://doi.org/10.1007/978-1-4939-1384-8_8

S. Kim and J. Ryu. 2011a. The sample average approximation method for multi-objective stochastic optimization. In Proceedings of the 2011 Winter Simulation Conference, S. Jain, R. R. Creasey, J. Himmelspach, K. P. White, and M. Fu (Eds.). IEEE, Piscataway, NJ, 4026-4037. DOI : https://doi.org/10.1109/WSC.2011.6148092

S. Kim and J. Ryu. 2011b. A trust-region algorithm for bi-objective stochastic optimization. Procedia Computer Science 4 (May 2011), 1422-1430. DOI : https://doi.org/10.1016/j.procs.2011.04.153

S.-H. Kim and B. L. Nelson. 2006. Selecting the best system. In Simulation, S. G. Henderson and B. L. Nelson (Eds.). Elsevier, Amsterdam, The Netherlands, 501-534.

G. Kirlik and S. Sayin. 2013. Computing the nadir point for multiobjective discrete optimization problems. Fournal of Global Optimization 62 (2013), 79-99. DOI : https://doi.org/10.1007/s10980-013-9939-0

T. Klein, A. Holzkämper, P. Calanca, R. Seppelt, and J. Fuhrer. 2013. Adapting agricultural land management to climate change: A regional multi-objective optimization approach. Landscape Ecology 28 (2013), 2029-2047. DOI : https://doi. org/10.1007/s10898-014-0227-6

T. C. Koopmans. 1951. Analysis of production as an efficient combination of activities. In Activity Analysis of Production and Allocation, T. C. Koopmans (Ed.). John Wiley \& Sons, New York, 33-97.

H. W. Kuhn and A. W. Tucker. 1951. Nonlinear programming. In Proceedings of the 2nd Berkeley Symposium on Mathematical Statistics and Probability, J. Neyman (Ed.). University of California Press, Berkeley, CA, 481-492. Retrieved from https:// projecteuclid.org/euclid.bsmsp/1200500249.

G. W. Kuriger and F. H. Grant. 2011. A lexicographic Nelder-Mead simulation optimization method to solve multi-criteria problems. Computers \& Industrial Engineering 60, 4 (2011), 555-565.

H. Kushner and G. G. Yin. 2003. Stochastic Approximation and Recursive Algorithms and Applications (2nd ed.). Stochastic Modeling and Applied Probability, Vol. 35. Springer-Verlag, New York. DOI : https://doi.org/10.1007/b97441

R. Lacour, K. Klamroth, and C. M. Fonseca. 2017. A box decomposition algorithm to compute the hypervolume indicator. Computers \& Operations Research 79 (March 2017), 347-360. DOI : https://doi.org/10.1016/j.cor.2016.06.021

A. M. Law. 2015. Simulation Modeling and Analysis (5th ed.). McGraw Hill Education, New York.

J. S. Lee. 2014. Advances in Simulation: Validity and Efficiency. Ph.D. Dissertation. Georgia Institute of Technology. Retrieved from http://hdl.handle.net/1853/53457.

L. H. Lee, E. P. Chew, S. Teng, and Y. Chen. 2008. Multi-objective simulation-based evolutionary algorithm for an aircraft spare parts allocation problem. European fournal of Operational Research 189, 2 (2008), 476-491.

L. H. Lee, E. P. Chew, S. Teng, and D. Goldsman. 2010. Finding the non-dominated Pareto set for multi-objective simulation models. IIE Transactions 42 (2010), 656-674. DOI : https://doi.org/10.1080/07408171003705367

L. H. Lee, E. P. Chew, S. Teng, and J. Li. 2009. Application of evolutionary algorithms for solving multi-objective simulation optimization problems. In Multi-objective Memetic Algorithms, C.-K. Goh, Y.-S. Ong, and K. C. Tan (Eds.). Springer, Berlin, 91-110.

L. H. Lee, C. U. Lee, and Y. P. Tan. 2007. A multi-objective genetic algorithm for robust flight scheduling using simulation. European fournal of Operational Research 177, 3 (2007), 1948-1968. DOI : https://doi.org/10.1016/j.ejor.2005.12.014

H. Li, L. H. Lee, E. P. Chew, and P. Lendermann. 2015a. MO-COMPASS: A fast convergent search algorithm for multiobjective discrete optimization via simulation. IIE Transactions 47, 11 (2015), 1153-1169. DOI : https://doi.org/10.1080/ 0740817X.2015.1005778 
H. Li, Y. Li, G. Pedrielli, L. H. Lee, E. P. Chew, and C.-H. Chen. 2015b. Multi-objective multi-fidelity optimization with ordinal transformation and optimal sampling. In Proceedings of the 2015 Winter Simulation Conference, L. Yilmaz, W. K. V. Chan, T. M. K. Roeder, C. Macal, and M. D. Rosetti (Eds.). IEEE, Piscataway, NJ, 3737-3747.

H. Li, G. Pedrielli, L. H. Lee, and E. P. Chew. 2017. Enhancement of supply chain resilience through inter-echelon information sharing. Flexible Services and Manufacturing fournal 29, 2 (2017), 260-285. DOI:https://doi.org/10.1007/ s10696-016-9249-3

H. Li, Y. Zhu, Y. Chen, G. Pedrielli, N. A. Pujowidianto, and Y. Chen. 2015c. The object-oriented discrete event simulation modeling: A case study on aircraft spare part management. In Proceedings of the 2015 Winter Simulation Conference, $\mathrm{L}$. Yilmaz, W. K. V. Chan, T. M. K. Roeder, C. Macal, and M.D. Rosetti (Eds.). IEEE, Piscataway, NJ, 3514-3525.

J. Li, W. Liu, G. Pedrielli, L. H. Lee, and E. P. Chew. 2018. Optimal computing budget allocation to select the non-dominated systems - A large deviations perspective. IEEE Transactions on Automatic Control (2018). DOI : https://doi.org/10.1109/ TAC.2017.2779603

R.-C. Lin, M. Y. Sir, and K. S. Pasupathy. 2013. Multi-objective simulation optimization using data envelopment analysis and genetic algorithm: Specific application to determining optimal resource levels in surgical services. Omega 41 (2013), 881-892. DOI : https://doi.org/10.1016/j.omega.2012.11.003

W. Liu, S. Gao, and L. H. Lee. 2017. A multi-objective perspective on robust ranking and selection. In Proceedings of the 2017 Winter Simulation Conference, W. K. V. Chan, A. D’Ambrogio, G. Zacharewicz, N. Mustafee, G. Wainer, and E. Page (Eds.). IEEE, Piscataway, NJ, 2116-2127. DOI : https://doi.org/10.1109/WSC.2017.8247944

S. Lucidi, M. Maurici, L. Paulon, F. Rinaldi, and M. Roma. 2016. A simulation-based multi-objective optimization approach for health care service management. IEEE Transactions on Automation Science and Engineering 13, 4 (2016), 1480-1491. DOI : https://doi.org/10.1109/TASE.2016.2574950

M. Mahdavi, T. Yang, and R. Jin. 2013. Stochastic convex optimization with multiple objectives. In Advances in Neural Information Processing Systems 26, C. J. C. Burges, L. Bottou, M. Welling, Z. Ghahramani, and K. Q. Weinberger (Eds.). Curran Associates, Red Hook, NY, 1115-1123. Retrieved from http://papers.nips.cc/paper/ 4942-stochastic-convex-optimization-with-multiple-objectives.pdf.

R. T. Marler and J. S. Arora. 2004. Survey of multi-objective optimization methods for engineering. Structural and Multidisciplinary Optimization 26 (2004), 369-395. DOI : https://doi.org/10.1007/s00158-003-0368-6

V. Mattila and K. Virtanen. 2014. Maintenance scheduling of a fleet of fighter aircraft through multi-objective simulationoptimization. Simulation: Transactions of the Society for Modeling and Simulation International 90, 9 (2014), 1023-1040. DOI : https://doi.org/10.1177/0037549714540008

V. Mattila and K. Virtanen. 2015. Ranking and selection for multiple performance measures using incomplete preference information. European fournal of Operational Research 242 (2015), 568-579. DOI : https://doi.org/10.1016/j.ejor.2014.10. 028

J. R. W. Merrick, D. Morrice, and J. C. Butler. 2015. Using multiattribute utility theory to avoid bad outcomes by focusing on the best systems in ranking and selection. Journal of Simulation 9, 3 (2015), 238-248. DOI : https://doi.org/10.1057/ jos.2014.34

A. Messac, A. Ismail-Yahaya, and C. A. Mattson. 2003. The normalized normal constraint method for generating the Pareto frontier. Structural and Multidisciplinary Optimization 25, 2 (2003), 86-98.

K. Miettinen. 1999. Nonlinear Multiobjective Optimization. Kluwer Academic Publishers, Boston.

B. L. Nelson. 2010. Optimization via simulation over discrete decision variables. In TutORials in Operations Research, J. J. Hasenbein, P. Gray, and H. J. Greenberg (Eds.). INFORMS, Catonsville, MD, Chapter 9, 193-207. DOI : https://doi.org/10. 1287/educ. 1100.0069

A. Nguyen, S. Reiter, and P. Rigo. 2014. A review on simulation-based optimization methods applied to building performance analysis. Applied Energy 113 (2014), 1043-1058. Retrieved from http://dx.doi.org/10.1016/j.apenergy.2013.08.061.

S. Ólafsson. 2006. Metaheuristics. In Simulation, S. G. Henderson and B. L. Nelson (Eds.). Elsevier, Amsterdam, The Netherlands, 633-654.

L. Pang, F. Meng, and J. Wang. 2018. Asymptotic convergence of stationary points of stochastic multiobjective programs with parametric variational inequality constraint via SAA approach. fournal of Industrial and Management Optimization (2018). DOI : https://doi.org/10.3934/jimo.2018116

A. Pascoletti and P. Serafini. 1984. Scalarizing vector optimization problems. fournal of Optimization Theory and Applications 42, 4 (1984), 499-524. DOI : https://doi.org/10.1007/BF00934564

R. Pasupathy and S. Ghosh. 2013. Simulation optimization: A concise overview and implementation guide. In TutORials in Operations Research, H. Topaloglu (Ed.). INFORMS, Catonsville, MD, Chapter 7, 122-150. DOI : https://doi.org/10.1287/ educ. 2013.0118

R. Pasupathy, P. W. Glynn, S. Ghosh, and F. Hashemi. 2018. On sampling rates in simulation-based recursions. SIAM fournal on Optimization 28, 1 (2018), 45-73. DOI : https://doi.org/10.1137/140951679 
R. Pasupathy and S. G. Henderson. 2006. A testbed of simulation-optimization problems. In Proceedings of the 2006 Winter Simulation Conference, L. F. Perrone, F. P. Wieland, J. Liu, B. G. Lawson, D. M. Nicol, and R. M. Fujimoto (Eds.). IEEE, Piscataway, NJ, 255-263. DOI : https://doi.org/10.1109/WSC.2006.323081

R. Pasupathy and S. G. Henderson. 2011. SimOpt: A library of simulation optimization problems. In Proceedings of the 2011 Winter Simulation Conference, S. Jain, R. R. Creasey, J. Himmelspach, K. P. White, and M. Fu (Eds.). IEEE, Piscataway, NJ, 4075-4085. DOI : https://doi.org/10.1109/WSC.2011.6148097

R. Pasupathy, S. R. Hunter, N. A. Pujowidianto, L. H. Lee, and C.-H. Chen. 2015. Stochastically constrained ranking and selection via SCORE. ACM Transactions on Modeling and Computer Simulation 25, 1, Article 1 (January 2015), 26 pages. DOI : https://doi.org/10.1145/2630066

R. Pasupathy and S. Kim. 2011. The stochastic root-finding problem: Overview, solutions, and open questions. ACM Transactions on Modeling and Computer Simulation 21, 3, Article 19 (2011), 23 pages. DOI: https://doi.org/10.1145/1921598. 1921603

R. Pasupathy and B. W. Schmeiser. 2010. Root finding via DARTS: Dynamic adaptive random target shooting. In Proceedings of the 2010 Winter Simulation Conference, B. Johansson, S. Jain, J. Montoya-Torres, J. Hugan, and E. Yücesan (Eds.). IEEE, Piscataway, NJ, 1255-1262. Retrieved from http://www.informs-sim.org/wsc10papers/115.pdf.

F. Poirion, Q. Mercier, and J.-A. Désidéri. 2017. Descent algorithm for nonsmooth stochastic multiobjective optimization. Computational Optimization and Applications 68, 2 (November 2017 2017), 317-331. DOI : https://doi.org/10.1007/ s10589-017-9921-x

O. Prakash, K. Srinivasan, and K. P. Sudheer. 2015. Adaptive multi-objective simulation-optimization framework for dynamic flood control operation in a river-reservoir system. Hydrology Research 46, 6 (2015), 893-911. DOI : https: //doi.org/10.2166/nh.2015.171

P. Reverdy. 2016. Gaussian multi-armed bandit problems with multiple objectives. In American Control Conference (ACC'16). IEEE, Piscataway, NJ, 5263-5269. DOI : https://doi.org/10.1109/ACC.2016.7526494

S. L. Rosen, C. M. Harmonosky, and M. T. Traband. 2007. A simulation optimization method that considers uncertainty and multiple performance measures. European fournal of Operational Research 181, 1 (August 2007), 315-330. DOI: https: //doi.org/10.1016/j.ejor.2006.05.040

S. L. Rosen, C. M. Harmonosky, and M. T. Traband. 2008. Optimization of systems with multiple performance measures via simulation: Survey and recommendations. Computers \& Industrial Engineering 54, 2 (March 2008), 327-339. DOI : https: //doi.org/10.1016/j.cie.2007.07.004

R. E. Rosenthal. 1985. Principles of multiobjective optimization. Decision Sciences 16 (1985), 133-152.

A. Ruszczyński and A. Shapiro (Eds.). 2003. Stochastic Programming. Elsevier, New York.

J. Ryu and S. Kim. 2014. A derivative-free trust-region method for biobjective optimization. SIAM fournal on Optimization 24, 1 (2014), 334-362. DOI : https://doi.org/10.1137/120864738

S. Sayin. 2000. Measuring the quality of discrete representations of efficient sets in multiple objective mathematical programming. Mathematical Programming, Series A 87 (2000), 543-560. DOI : https://doi.org/10.1007/s101070050011

B. Schmeiser. 2008. A practitioner, a vender, and a researcher walk into a bar: Trying to explain what researchers do. In Proceedings of the 2008 Winter Simulation Conference, S. J. Mason, R. R. Hill, L. Mönch, O. Rose, T. Jefferson, and J. W. Fowler (Eds.). IEEE, Piscataway, NJ, 2-9. DOI : https://doi.org/10.1109/WSC.2008.4736049

A. Shapiro. 2003. Monte Carlo sampling methods. In Stochastic Programming, A. Ruszczyński and A. Shaprio (Eds.). Elsevier, Amsterdam, The Netherlands, 353-425.

A. Shapiro, D. Dentcheva, and A. Ruszczyński. 2009. Lectures on Stochastic Programming: Modeling and Theory. Society for Industrial and Applied Mathematics, Philadelphia, PA.

S. Shashaani, F. S. Hashemi, and R. Pasupathy. 2018. ASTRO-DF: A class of adaptive sampling trust-region algorithms for derivative-free simulation optimization. SIAM fournal on Optimization 28, 4 (2018), 3145-3176. Retrieved from https:// arxiv.org/pdf/1610.06506v1.pdf.

F. Siegmund, A. H. C. Ng, and K. Deb. 2016. Hybrid dynamic resampling algorithms for evolutionary multi-objective optimization of invariant-noise problems. In Applications of Evolutionary Computation: 19th European Conference, EvoApplications, Part II, G. Squillero and P. Burelli et al. (Eds.). Springer, New York, 311-216. DOI : https://doi.org/10.1007/ 978-3-319-31153-1 21

A. Singh and B. S. Minsker. 2008. Uncertainty-based multiobjective optimization of groundwater remediation design. Water Resources Research 44, Article W02404 (2008), 24 pages. DOI : https://doi.org/10.1029/2005WR004436

A. Singhee and P. Castalino. 2010. Pareto sampling: Choosing the right weights by derivative pursuit. In Proceedings of the 47th Design Automation Conference. ACM, New York, 913-916.

J. Song, Y. Qiu, and Z. Liu. 2016. Integrating optimal simulation budget allocation and genetic algorithm to find the approximate Pareto patient flow distribution. IEEE Transactions on Automation Science and Engineering 13, 1 (2016), 149-159. DOI : https://doi.org/10.1109/TASE.2015.2424975 
W. Stadler. 1979. A survey of multicriteria optimization or the vector maximization problem, part I: 1776-1960. fournal of Optimization Theory and Applications 29, 1 (September 1979), 1-52.

I. Steponavičè, S. Ruuska, and K. Miettinen. 2014. A solution process for simulation-based multiobjective design optimization with an application in the paper industry. Computer-Aided Design 47 (2014), 45-58. DOI: https://doi.org/10.1016/j. cad.2013.08.045

K. Subramanyan, U. Diwekar, and S. E. Zitney. 2011. Stochastic modeling and multi-objective optimization for the APECS system. Computers and Chemical Engineering 35 (2011), 2667-2679. DOI : https://doi.org/10.1016/j.compchemeng.2011. 02.003

E. Tekin and I. Sabuncuoglu. 2004. Simulation optimization: A comprehensive review on theory and applications. IIE Transactions 36, 11 (2004), 1067-1081. DOI : https://doi.org/10.1080/07408170490500654

S. Teng, L. H. Lee, and E. P. Chew. 2010. Integration of indifference-zone with multi-objective computing budget allocation. European fournal of Operational Research 203, 2 (2010), 419-429. DOI : https://doi.org/10.1016/j.ejor.2009.08.008

D. Vanderpooten, L. Weerasena, and M. M. Wiecek. 2017. Covers and approximations in multiobjective optimization. fournal of Global Optimizaiton 67, 3 (March 2017), 601-619. DOI : https://doi.org/10.1007/s10898-016-0426-4

M. G. Villarreal-Marroquín, J. M. Castro, and M. Cabrera-Ríos. 2011. A multicriteria simulation optimization method for injection molding. In Proceedings of the 2011 Winter Simulation Conference, S. Jain, R. R. Creasey, J. Himmelspach, K. P. White, and M. Fu (Eds.). IEEE, Piscataway, NJ, 2395-2407.

M. G. Villarreal-Marroquín, J. D. Svenson, F. Sun, T. J. Santner, A. Dean, and J. M. Castro. 2013. A comparison of two metamodel-based methodologies for multiple criteria simulation optimization using an injection molding case study. Journal of Polymer Engineering 33, 3 (2013), 193-209. DOI : https://doi.org/10.1515/polyeng-2013-0022

H. Wang. 2013. Zigzag search for continuous multiobjective optimization. INFORMS fournal on Computing 25, 4 (2013), 654-665. DOI : https://doi.org/10.1287/ijoc.1120.0528

H. Wang. 2017. Multi-objective retrospective optimization using stochastic zigzag search. European fournal of Operational Research 263, 3 (December 2017), 946-960. DOI : https://doi.org/10.1016/j.ejor.2017.06.039

H. Wang, R. Pasupathy, and B. W. Schmeiser. 2013. Integer-ordered simulation optimization using R-SPLINE: Retrospective search using piecewise-linear interpolation and neighborhood enumeration. ACM Transactions on Modeling and Computer Simulation 23, 3, Article 17 (2013), 24 pages. DOI : https://doi.org/10.1145/2499913.2499916

W. Wang and H. Wan. 2017. Sequential probability ratio test for multiple-objective ranking and selection. In Proceedings of the 2017 Winter Simulation Conference, W. K. V. Chan, A. D'Ambrogio, G. Zacharewicz, N. Mustafee, G. Wainer, and E. Page (Eds.). IEEE, Piscataway, NJ, 1998-2009. DOI : https://doi.org/10.1109/WSC.2017.8247934

Y. Wang, L. H. Lee, E. P. Chew, S. S. W. Lam, S. K. Low, M. E. H. Ong, and H. Li. 2015. Multi-objective optimization for a hospital inpatient flow process via discrete event simulation. In Proceedings of the 2015 Winter Simulation Conference, L. Yilmaz, W. K. V. Chan, T. M. K. Roeder, C. Macal, and M. D. Rosetti (Eds.). IEEE, Piscataway, NJ, 3622-3631.

D. J. White. 1990. A bibliography on the applications of mathematical programming multiple-objective methods. fournal of the Operational Research Society 41, 8 (1990), 669-691.

M. M. Wiecek, M. Ehrgott, and A. Engau. 2016. Continuous multiobjective programming. In Multiple Criteria Decision Analysis: State of the Art Surveys, S. Greco, M. Ehrgott, and J. R. Figueira (Eds.). International Series in Operations Research \& Management Science, Vol. 233. Springer New York, New York, 739-815. DOI : https://doi.org/10.1007/ 978-1-4939-3094-4_18

J. Wu and S. Azarm. 2001. Metrics for quality assessment of a multiobjective design optimization solution set. Transactions of the ASME 123 (2001), 18-25. DOI : https://doi.org/10.1115/1.1329875

J. Xu, E. Huang, C.-H. Chen, and L. H. Lee. 2015. Simulation optimization: A review and exploration in the new era of cloud computing and big data. Asia-Pacific Journal of Operational Research 32, 3, Article 1550019 (2015), 34 pages. DOI : https: //doi.org/10.1142/S0217595915500190

J. Xu, B. L. Nelson, and L. J. Hong. 2010. Industrial strength COMPASS: A comprehensive algorithm and software for optimization via simulation. ACM Transactions on Modeling and Computer Simulation 20 (2010), 1-29.

S. Q. Yahyaa and M. M. Drugan. 2015. Correlated Gaussian multi-objective multi-armed bandit across arms algorithm. In IEEE Symposium series on Computational Intelligence. IEEE, Piscataway, NJ, 593-600. DOI : https://doi.org/10.1109/SSCI 2015.93

S. Q. Yahyaa, M. M. Drugan, and B. Manderick. 2014a. Knowledge gradient for multi-objective multi-armed bandit algorithms. In 2014 International Conference on Agents and Artificial Intelligence (ICAART'14). SCITEPRESS, Setubal, Portugal, 74-83.

S. Q. Yahyaa, M. M. Drugan, and B. Manderick. 2014b. Multivariate normal distribution based multi-armed bandit Pareto algorithm. In The 7th European Conference on Machine Learning and Principles and Practice of Knowledge and Discovery in Databases (EMCL/PKDD'14), PhD Track. Springer, New York, 139-148.

S. Q. Yahyaa, M. M. Drugan, and B. Manderick. 2014c. The scalarized multi-objective multi-armed bandit problem: An empirical study of its exploration vs. exploitation tradeoff. In 2014 International foint Conference on Neural Networks (IfCNN'14). IEEE, Piscataway, NJ, 2290-2297. DOI : https://doi.org/10.1109/IJCNN.2014.6889390 
S. Q. Yahyaa, M. M. Drugan, and B. Manderick. 2015. Thompson sampling in the adaptive linear scalarized multi objective multi armed bandit. In Proceedings of the International Conference on Agents and Artificial Intelligence (ICAART'15), Vol. 2. SCITEPRESS, Portugal, 55-65. DOI : https://doi.org/10.5220/0005184400550065

H. Yildiz and S. Suri. 2012. On Klee's measure problem for grounded boxes. In Proceedings of the 28th Annual Symposium on Computational Geometry. ACM, New York, 111-1120. DOI : https://doi.org/10.1145/2261250.2261267

M. Yoon and J. Bekker. 2017. Single- and multi-objective ranking and selection procedures in simulation: A historical review. South African fournal of Industrial Engineering 28, 2 (2017), 37-45. DOI : https://doi.org/10.7166/28-2-1732

H. Zhang. 2008. Multi-objective simulation-optimization for earthmoving operations. Automation in Construction 18 (2008), 79-86. DOI : https://doi.org/10.1016/j.autcon.2008.05.0023

J. Zhang, Y. Ma, T. Yang, and L. Liu. 2017. Estimation of the Pareto front in stochastic simulation through stochastic Kriging. Simulation Modelling Practice and Theory 79 (2017), 69-86. DOI : https://doi.org/10.1016/j.simpat.2017.09.006

A. Zhou, B. Qu, H. Li, S. Zhao, P. N. Suganthan, and Q. Zhang. 2011. Multiobjective evolutionary algorithms: A survey of the state of the art. Swarm and Evolutionary Computation 1 (2011), 32-49.

C. Zhou, H. Li, B. K. Lee, and Z. Qiu. 2018. A simulation-based vessel-truck coordination strategy for lighterage terminals. Transportation Research Part C: Emerging Technologies 95 (October 2018), 149-164. DOI : https://doi.org/10.1016/j.trc.2018. 07.015

E. Zitzler. 1999. Evolutionary Algorithms for Multiobjective Optimization: Methods and Applications. Ph.D. Dissertation. Swiss Federal Institute of Technology Zurich.

E. Zitzler, K. Deb, and L. Thiele. 2000. Comparison of multiobjective evolutionary algorithms: Empirical results. Evolutionary Computation 8, 2 (2000), 173-195.

E. Zitzler, L. Thiele, M. Laumanns, C. M. Fonseca, and V. Grunert da Fonseca. 2003. Performance assessment of multiobjective optimizers: An analysis and review. IEEE Transactions on Evolutionary Computation 7, 2 (2003), 117-132. DOI : https://doi.org/10.1109/TEVC.2003.810758

Received March 2017; revised August 2018; accepted November 2018 